\title{
Topology Optimization of Multi-Material Negative Poisson's Ratio Metamaterials Using a Reconciled Level Set Method
}

\author{
Panagiotis Vogiatzis, Shikui Chen*, Xiao Wang , Tiantian Li and Lifeng Wang \\ Department of Mechanical Engineering, State University of New York at Stony Brook, Stony Brook, New York 11794, USA
} Email: Shikui.Chen@stonybrook.edu

Metamaterials are defined as a family of rationally designed artificial materials which can provide extraordinary effective properties compared with their nature counterparts. This paper proposes a level set based method for topology optimization of both single and multiple-material Negative Poisson's Ratio (NPR) metamaterials. For multi-material topology optimization, the conventional level set method is advanced with a new approach exploiting the reconciled level set (RLS) method. The proposed method simplifies the multi-material topology optimization by evolving each individual material with a single level set function and reconciling the result level set field with the Merriman-Bence-Osher (MBO) operator. The NPR metamaterial design problem is recast as a variational problem, where the effective elastic properties of the spatially periodic microstructure are formulated as the strain energy functionals under uniform displacement boundary conditions. The adjoint variable method is utilized to derive the shape sensitivities by combining the general linear elastic equation with a weak imposition of Dirichlet boundary conditions. The design velocity field is constructed using the steepest descent method and integrated with the level set method. Both single and multiple-material mechanical metamaterials are achieved in 2D and 3D with different Poisson's ratios and volumes. Benchmark designs are fabricated with multi-material 3D printing at high resolution. The effective auxetic properties of the achieved designs are verified through finite element simulations and characterized using experimental tests as well.

Keywords: Topology optimization, Multi-material, Negative Poisson's Ratio, Metamaterial, Reconciled Level Set Method

\section{INTRODUCTION}

Metamaterials gain extraordinary effective properties from rationally designed structures rather than their composition, and thus the layout of the microstructure has a significant impact on their properties. This paper focuses on designing mechanical metamaterials with negative Poisson's ratios (NPR), which are also known as auxetics or auxetic materials. When an NPR material is compressed along a particular axis, it is observed to contract in the perpendicular directions, as demonstrated in Figure 1. This counter-intuitive property can be utilized to strengthen mechanical properties for the purpose of improving the crack resistance [1], increasing the fracture toughness [2, 3], or providing higher sound absorption capacity [4]. Since Lakes first developed NPR foam structures in 1987 [5], the research work on NPR metamaterials modeling, design and manufacturing as well as their potential applications has advanced considerably [6-14]. In 1985, Kolpakov proposed a method for approximating the average elastic characteristics of framework structures of periodic configuration, and constructed fine-celled framework structures with negative Poisson's ratios [15]. Almgren presented a strategy to make a structure of rods, hinges and springs to achieve a negative Poisson's ratio equal to -1 [16]. Theocaris et al. employed the numerical homogenization theory for the investigation of composite structures with star-shaped inclusions, which are able to exhibit a negative Poisson's ratio [17].

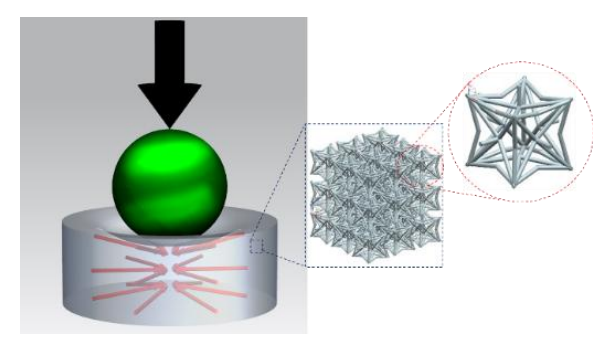

FIG. 1. 3D auxetic mechanical metamaterial with a negative Poisson's ratio

*Address all responses to this author.

Conventional design practice for metamaterials relies heavily on 
designers' intuition or analogy to existing design solutions, which is limited by a small design space. Topology optimization (TO) [18] is a powerful computational tool which recasts the design problem as an optimal material distribution problem so that the system will find an optimal material layout to fulfill the functional requirements quantified by the objective and constraint functions in the optimization scheme. During the past three decades, topology optimization of lightweight load-carrying structures with linear elastic behaviors has been well established [19-21], and was naturally extended to systematical design of metamaterials with highly nonlinear behavior and multi-physics coupling characteristics. To the authors' best knowledge, the first engineered microstructures with prescribed constitutive parameters was designed by Sigmund using inverse homogenization theory in 1994 [22]. Sigmund [23] successfully applied inverse homogenization to obtain truss frame microstructures with negative Poisson's ratios both in 2D and 3D. Wang et al. [24] and Allaire et al. [25] both applied a level-set based optimization method to 2D NPR designs. Zhang et al. [26] employed the strain energy-based method to predict effective elastic properties and applied a dual optimization algorithm to the design of microstructure with tailored elastic properties. Even though 2D NPR designs can be extruded in order to get manufacturable 3D geometries [27], interests in 3D microstructures have led to some recent developments in NPR structure design. Schwerdtfeger et al. [28] using solid isotropic material with penalization (SIMP) method obtained a 3D NPR microstructure. In another recent contribution, Andreassen et al. [29] designed a manufacturable 3D extremal elastic microstructure using a modified SIMP method. More recently, Shan et al. [30] developed 2D isotropic NPR structures by embedding periodic arrays of cuts in elastomeric sheets. Moreover, Clausen et al. [31] designed microstructures with programmable Poisson's ratios over large deformations.

This paper is concerned with an improved level set method for the rational design of 2D and 3D NPR metamaterials, multi-phase NPR metamaterials. Level set methods, originally developed for tracking free fluid boundaries [32], have grown to be an attractive tool for topology optimization [33, 34]. The key idea of the level set methods is to implicitly represent a moving boundary as the zero level set of a function with one higher dimension. The motion of the boundary is numerically described using the Hamilton-Jacobi partial differential equation (H-J PDE) [32]. The design velocity field, which is usually obtained via shape sensitivity analysis, will drive the boundary to evolve during the design process. Further technical details about the level set method will be provided in Section 3.

The paper begins by explaining how to evaluate the effective properties of the unit cell with the strain energy-based method. The problem settings for designing NPR microstructures is provided in section 3, followed by numerical examples to demonstrate the performance of the proposed method in Section 4. In section 5, auxetic effects are verified through finite element simulations and validated through experimental tests with 2D NPR metamaterials. Concluding remarks are provided in section 6 .

\section{PREDICTION OF EFFECTIVE ELASTIC MODULUS TENSORS OF A UNIT CELL}

Metamaterials are characterized by the spatially periodic arrangement of the microstructures, as shown in Figure 2. The effective elastic properties of a continuous medium directly depend on its microstructure. A key issue here is how to link field variables at microscopic length scale to field variables at macroscopic length scale. In micromechanics, this relation can be formulated using two basic theories: the homogenization theory [35-42] and the strain energy based method [26]. A quantitative comparison of the two methods was provided by Hollister and Kikuchi [43]. 


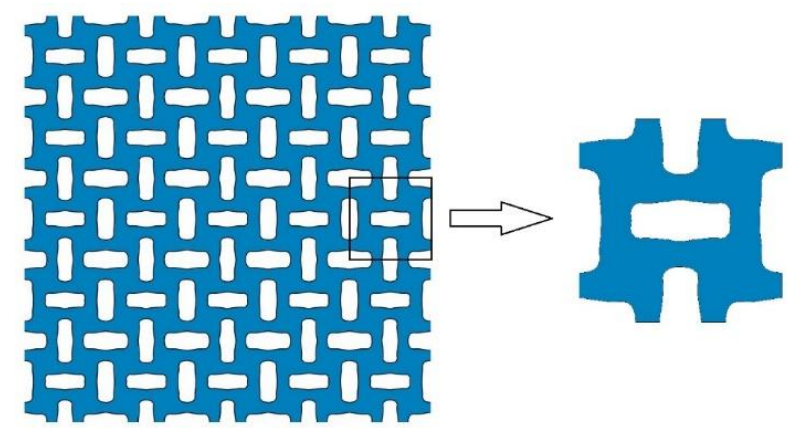

FIG. 2. Periodically assembled unit cell

\section{Strain Energy-Based Method}

The strain energy-based method originates from the standard mechanics analysis, which formulates the effective modulus tensors of the unit cell as the strain energy functionals under different boundary conditions. Compared with the homogenization theory, the strain energy-based method provides a relatively simple and efficient way [26] in the estimation of the homogenized properties and the sensitivity analysis as well. There are three loading cases for $2 \mathrm{D}$ problems with $k l=11,22,12$ and six loading cases for $3 \mathrm{D}$ problems with $k l=11,22,33,12,23,13$.

For linear elastic problems, the average stress and average strain of the homogeneous medium are equivalent to the domain integration of the local stress and local strain in the microstructure, which can be mathematically expressed as $\bar{\sigma}_{i j}=\frac{1}{V} \int \sigma_{i j} d V$ and $\bar{\varepsilon}_{k l}=\frac{1}{V} \int \varepsilon_{k l} d V$. The constitutive law between average stress and average strain can be characterized by the generalized Hooke's Law as:

$$
\overline{\boldsymbol{\sigma}}_{i j}=\mathbf{C}_{i j k l}^{H} \overline{\boldsymbol{\varepsilon}}_{k l}
$$

In the above equation, $\mathbf{C}_{i j k l}^{H}$ is the homogenized elastic modulus tensor, which depends on the layout of the materials of the unit cell. For a 2D orthotropic medium, equation (1) can be rewritten as follows:

$$
\left[\begin{array}{l}
\bar{\sigma}_{11} \\
\bar{\sigma}_{22} \\
\bar{\sigma}_{12}
\end{array}\right]=\left[\begin{array}{ccc}
C_{1111}^{H} & C_{1122}^{H} & 0 \\
C_{1122}^{H} & C_{2222}^{H} & 0 \\
0 & 0 & C_{1212}^{H}
\end{array}\right]\left[\begin{array}{l}
\bar{\varepsilon}_{11} \\
\bar{\varepsilon}_{22} \\
- \\
\bar{\varepsilon}_{12}
\end{array}\right]
$$

The multiscale structure-property relation can be constructed according to the fact that the strain energy of the homogeneous medium, which is defined as $U^{H}=\frac{1}{2} V \bar{\sigma}_{i j} \cdot \bar{\varepsilon}_{k l}$, and the strain energy of the unit cell, which is defined as $U=\frac{1}{2} \int_{V} \sigma_{i j} \varepsilon_{k l} d V$, are equivalent. This multiscale structure-property relation can be formulated as follows:

$$
\frac{1}{2} V \bar{\sigma}_{i j} \cdot \bar{\varepsilon}_{k l}=\frac{1}{2} \int_{V} \sigma_{i j} \varepsilon_{k l} d V .
$$

Substitute equation (1) into equation (3), the effective elastic stiffness constants $C_{1111}^{H}, C_{2222}^{H}$ and $C_{1122}^{H}$ can be determined by applying the following three unit strain fields respectively:

$$
\overline{\boldsymbol{\varepsilon}}_{(11)}=\left\{\begin{array}{l}
1 \\
0 \\
0
\end{array}\right\}, \overline{\boldsymbol{\varepsilon}}_{(22)}=\left\{\begin{array}{l}
0 \\
1 \\
0
\end{array}\right\}, \overline{\boldsymbol{\varepsilon}}_{(1122)}=\left\{\begin{array}{l}
1 \\
1 \\
0
\end{array}\right\} .
$$


The uniform strain boundary condition is replaced with equivalent Dirichlet boundary condition in numerical implementation. For example, the effective elastic stiffness constant $C_{1111}^{H}$ can be derived from loading case 1, shown in Figure 3(a), with the corresponding Dirichlet boundary conditions as follow:

$$
\begin{aligned}
& u_{1}=1, v_{2}=v_{4}=0, u_{3}=0 \\
U_{1111}^{H}= & \frac{1}{2} V\left[\begin{array}{l}
1 \\
0 \\
0
\end{array}\right]^{T}\left[\begin{array}{ccc}
C_{1111}^{H} & C_{1122}^{H} & 0 \\
C_{1122}^{H} & C_{2222}^{H} & 0 \\
0 & 0 & C_{1212}^{H}
\end{array}\right]\left[\begin{array}{l}
1 \\
0 \\
0
\end{array}\right] \\
= & \frac{1}{2} V C_{1111}^{H} .
\end{aligned}
$$

where th

The total strain energy can also be expressed using the domain integration of the local strain energy density in the unit cell, as equation (7) shows:

$$
U_{1111}=\frac{1}{2} \int_{\Omega} \boldsymbol{\varepsilon}_{11}^{T} \mathbf{D}_{i j k l} \boldsymbol{\varepsilon}_{11} d \Omega
$$

Assuming the volume of the unit cell is equal to 1 and keeping in mind that $U_{1111}^{H}=U_{1111}$, the expression of the effective elastic stiffness constant $C_{1111}^{H}$ can be further simplified to $C_{1111}^{H}=2 U_{1111}$, and similarly $C_{2222}^{H}=2 U_{2222}$. The effective elastic stiffness constant $C_{1122}^{H}$ can be formulated as a function of the strain energy by setting the boundary conditions as:

$$
u_{1}=1, v_{2}=1, u_{3}=0, v_{4}=0
$$

The corresponding strain energy becomes:

$$
\begin{aligned}
U_{1122}^{H} & =\frac{1}{2} V\left[\begin{array}{l}
1 \\
1 \\
0
\end{array}\right]^{T}\left[\begin{array}{ccc}
C_{1111}^{H} & C_{1122}^{H} & 0 \\
C_{1122}^{H} & C_{2222}^{H} & 0 \\
0 & 0 & C_{1212}^{H}
\end{array}\right]\left[\begin{array}{l}
1 \\
1 \\
0
\end{array}\right] \\
& =\frac{1}{2} V\left(C_{1111}^{H}+C_{1122}^{H}+C_{1122}^{H}+C_{2222}^{H}\right)
\end{aligned}
$$

By substituting $C_{1111}^{H}=2 U_{1111}$ and $C_{2222}^{H}=2 U_{2222}$ into equation (9), it can be derived that $C_{1122}^{H}=U_{1122}-U_{1111}-U_{2222}$. When it comes to $3 \mathrm{D}$ unit cells with orthotropic material, there are 9 independent elastic stiffness constants:

$$
C_{i j k l}=\left[\begin{array}{cccccc}
C_{1111} & C_{1122} & C_{1133} & 0 & 0 & 0 \\
C_{1122} & C_{2222} & C_{2233} & 0 & 0 & 0 \\
C_{1133} & C_{2233} & C_{3333} & 0 & 0 & 0 \\
0 & 0 & 0 & C_{2323} & 0 & 0 \\
0 & 0 & 0 & 0 & C_{1313} & 0 \\
0 & 0 & 0 & 0 & 0 & C_{1212}
\end{array}\right]
$$

The same calculations are made for the 3D case and the results are summarized in Table I. 
TABLE I. Effective elastic stiffness constants for 3D case

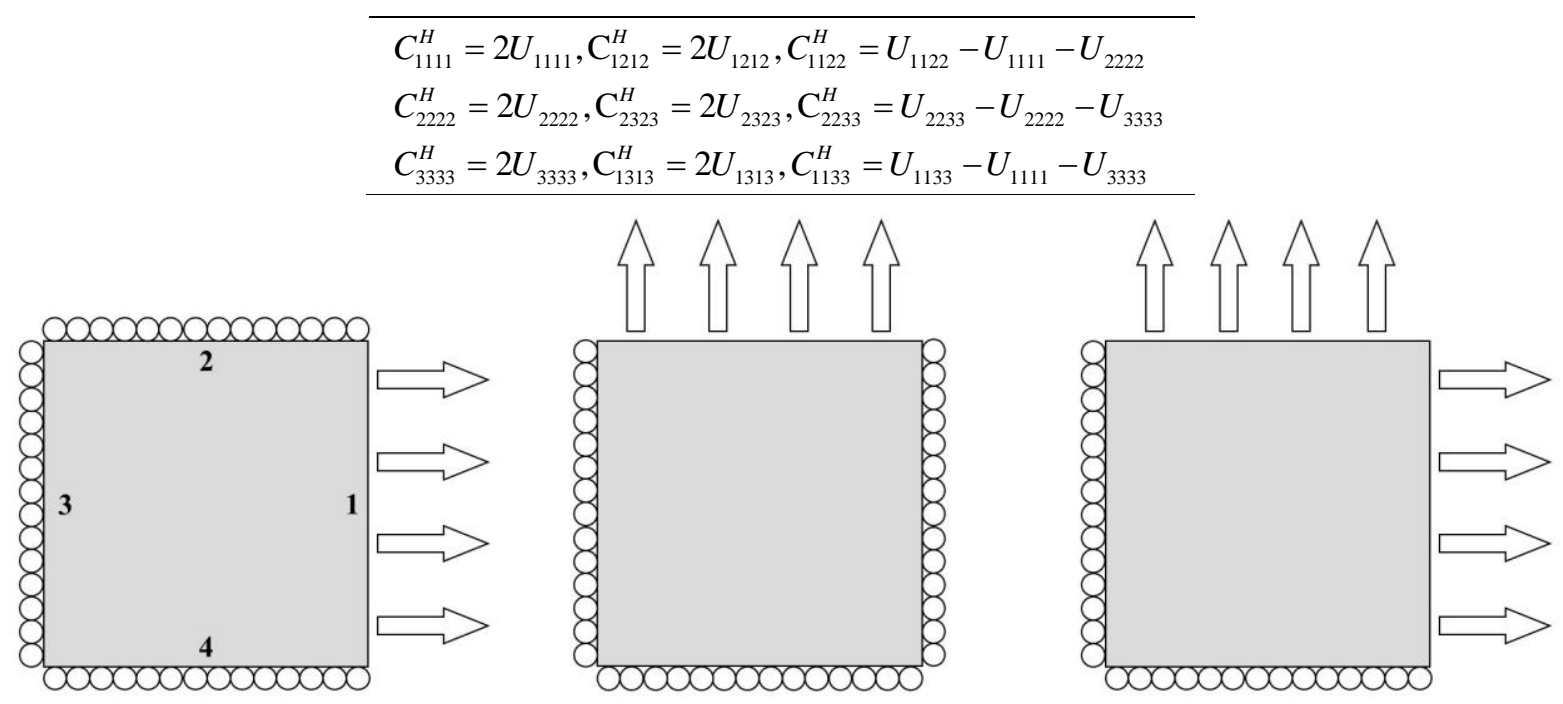

FIG. 3. Boundary conditions for unit cell: a) Loading case 1, b) Loading case 2, and c) Loading case 3.

\section{MULTI-PHASE TOPOLOGY OPTIMIZATION OF MICROSTRUCTURES}

\section{A. Level Set Methods}

Level set methods, introduced by Osher and Sethian [32], have become an attractive approach in topology optimization [33, 34], which provide smooth boundaries, crisp interfaces, high shape fidelity and great topological flexibility. Sethian and Wiengmann [44] employed level set approach along with immersed interface method for structural boundary design. Osher and Santosa [45] introduced a shape sensitivity analysis using gradient method to obtain a velocity field, and later Allaire et al. [34,46] proposed a method where the velocity field is derived from shape sensitivity analysis by employing the adjoint variable method. Wang et al. [47] established a link between the general structural optimization and the level set method through a design velocity field calculated using the material derivative in continuum mechanics. Belytschko et al. [48] developed a method with implicit function and regularization enabling sensitivity evaluations. Radial Basis Functions (RBFs) were proposed by Wang et al. [49-51] to parameterize the level set function and transform the Hamilton-Jacobi equation into a system of ordinary differential equations, which not only increased the efficiency of level set method but also improved its robustness in handling multiple constraints.

As its name implies, in level set methods, the boundary is implicitly represented by an isosurface of a surface (level set function) with one higher dimension, which divides the design domain into three parts according to the signs of the level set function, as shown in Figure 4.

$$
\begin{aligned}
& \phi(x(t), t)>0, \forall x \in \Omega(\text { material }) \\
& \phi(x(t), t)=0, \forall x \in \Gamma(\text { boundary }) \\
& \phi(x(t), t)<0, \forall x \in D \backslash \Omega(\text { void })
\end{aligned}
$$




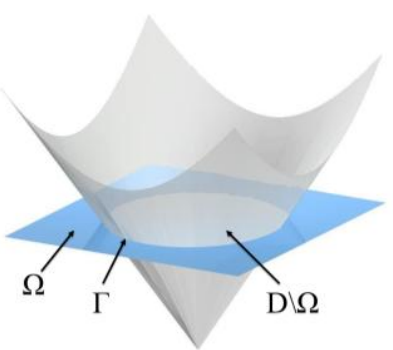

FIG. 4. Representation of a 2D geometry with a 3D level set function

By differentiating $\phi(x(t), t)=0$ with respect to the pseudo time, the Hamilton-Jacobi equation [32, 52] is obtained: $\frac{\partial \phi(x, t)}{\partial t}+\nabla \phi(x, t)$ where $\mathbf{V}(\mathrm{x})=d x / d t$ is the velocity field on the boundary. Equation (12) can be equivalently reformulated as:

$$
\frac{\partial \phi(x, t)}{\partial t}+V_{n}(\mathrm{x}) \nabla \phi(x, t)=0
$$

where $V_{n}(\mathrm{x})=V(\mathrm{x}) \cdot n=V(\mathrm{x}) \cdot \frac{\nabla \phi(x, t)}{|\nabla \phi(x, t)|}$ is the normal velocity field.

In this way, the optimization problem can be transformed into a problem of finding the steady-state solution of equation (13). The shape sensitivity analysis and the construction of the design velocity field is presented in subsection E.

\section{B. Reconciled Level Set Method for Multi-Material Shape and Topology Optimization}

Multi-material optimization schemes have been developed based on the classical single material optimization methods. Sigmund [53] employed SIMP with a modified interpolation scheme, based on Hashin-Shtrikman bounds, for a two-material Multiphysics actuator. Gao et al. [54, 55] proposed a multiphase optimization based on a mass constraint, instead of volume constraint. Wang and Wang [56] developed the 'Color' level set method (CLSM) [5658] which separates the materials by using the different sign combinations of $n$ level set functions. In this way, those $n$ level set functions can be utilized to represent at most $2^{n}$ materials. An alternative method is the Piecewise constant level set method [59-61], in which different values of the level set functions split the design domain into different areas. In this work, the Reconciled level set method is employed for topology optimization of NPR metamaterials. The RLSM was first introduced by Merriman, Bence and Osher [62, 63] for modeling multi-phase flow and was first employed by the author for multi-material topology optimization of smart energy harvesters [64]. RLSM retains the features of CLSM in multi-material representation and the convenience in the specification of arbitrary design velocities on each level set function. In addition, RLSM offers a more straightforward and convenient way to implement multi-material topology optimization than CLSM, since each individual material is uniquely represented by an independent level set function.

A critical issue in evolving multiple level set functions is that the independent evolution of the different level set functions may lead to overlaps or voids. In order to avoid such problems, the Merriman-Bence-Osher (MBO) operator $[62,63]$ is employed to reconcile the level set function of each material $i$ in relation to the rest of the materials $j$ :

$$
\phi_{i}^{1}=\frac{1}{2}\left(\phi_{i}^{t e m p}-\max _{i \neq j} \phi_{j}^{t e m p}\right)
$$

where $\phi_{i}^{\text {temp }}$ stands for the level set function after the evolution and the $\phi_{i}^{1}$ denotes the final level set function after applying the $\mathrm{MBO}$ operator. The subscripts $i$ and $j$ refer to the different materials. The RLSM is a predictor- 
corrector computational procedure. The impact of the $\mathrm{MBO}$ operator on the boundary evolution process is illustrated in Figure 5, which demonstrates the evolution process of two materials in two consecutive iterations, 0 and 1, with each individual material represented using a level set function independently. At iteration 0 , the circles are located in a distance of each other, as shown in Figure 5(a). Without the use of the MBO operator, the evolution of the independent level set functions may result in an overlap intersected between the two materials as shown in Figure 5(b). The MBO operator will correct the coupled level set functions, as shown in Figure 5(c). A comparison of the pros and cons between RLSM and CLSM is summarized in Table II.
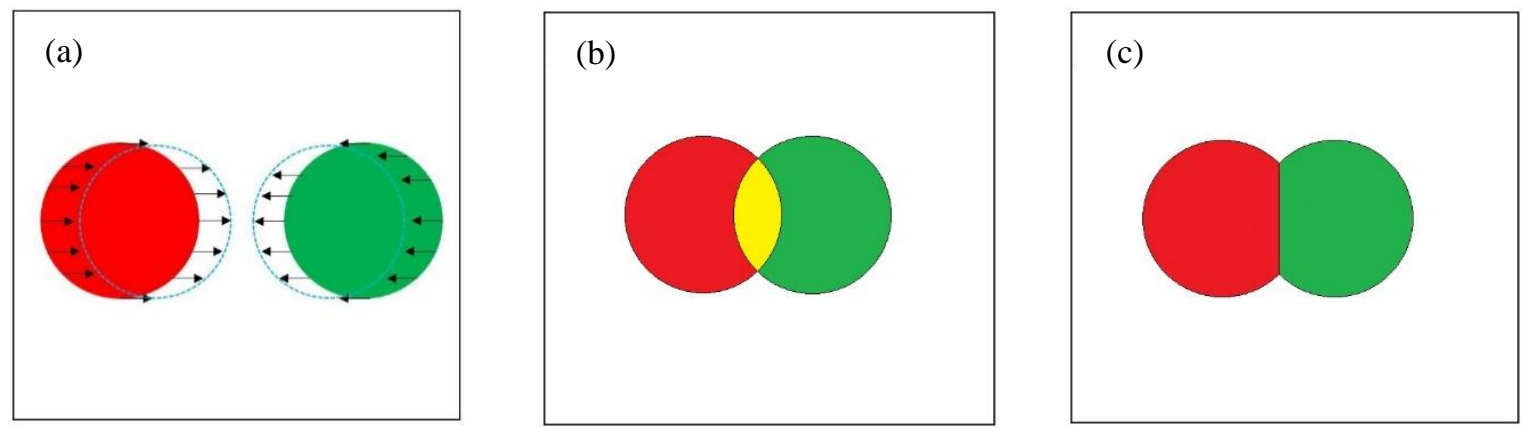

FIG. 5. Multi-material level set evolution. a) Before evolution (iteration 0); b) intermediate state (temp); c) with MBO operator applied (iteration 1).

TABLE II. Summary comparison between RLSM and CLSM

\begin{tabular}{|c|c|}
\hline Reconciled Level set method & 'Color' level set method \\
\hline $\begin{array}{l}\text { 1. } \mathrm{n} \text { level set functions represent } \mathrm{n} \text { material } \\
\text { phase }\end{array}$ & 1. $\mathrm{n}$ level set functions represent $2^{\mathrm{n}}$ material phase \\
\hline $\begin{array}{l}\text { 2. One-to-one relationship between level set } \\
\text { function and material }\end{array}$ & $\begin{array}{l}\text { 2. Different sign combinations of the level set } \\
\text { functions identify different materials }\end{array}$ \\
\hline 3. $\mathrm{MBO}$ operator to avoid overlaps & 3. No overlaps \\
\hline $\begin{array}{l}\text { 4. Straightforward and convenient for } \\
\text { implementation }\end{array}$ & 4. Not straightforward to construct initial design \\
\hline $\begin{array}{l}\text { 5. The level set function for each material is a } \\
\text { signed distance function }\end{array}$ & $\begin{array}{l}\text { 5. The combined level set function for each material } \\
\text { is no longer a signed distance function }\end{array}$ \\
\hline
\end{tabular}

\section{Problem Settings for NPR Metamaterial Design}

The NPR materials design problem can be formulated as a least square optimization problem to minimize the difference between the homogenized elastic stiffness constant $C_{i j k l}^{H}$ and the design targets $C_{i j k l}^{*}$ :

$$
\begin{array}{cl}
\text { Minimize } & J=\frac{1}{2} \sum_{i, j, k, l=1}^{d} w_{i j k l}\left(C_{i j k l}^{H}-C_{i j k l}^{*}\right)^{2} \\
\text { subject to: } & a(u, v)=l(v), \quad \forall v \in \bar{U}(Y) \\
& V(\Omega)=V^{*}
\end{array}
$$

where $\mathrm{d}$ is 2 for $2 \mathrm{D}$ and 3 for $3 \mathrm{D}$ studies, $w_{i j k l}$ is the weighting factor associated with the corresponding elasticity tensors, $V(\Omega)$ is the volume of the unit cell and $V^{*}$ is the volume target. The bilinear energy form $a(u, v)$, the linear load form $l(v)$ and the volume of the unit cell are described by: 


$$
\begin{aligned}
& a(u, v)=\int_{D} \varepsilon_{i j}^{T}(u) C_{i j k l} \varepsilon_{k l}(v) H(\phi) d \Omega \\
& l(v)=\int_{D} \varepsilon_{i j}^{0} C_{i j k l} \varepsilon_{k l}(v) H(\phi) d \Omega \\
& V(\Omega)=\int_{D} H(\phi) d \Omega
\end{aligned}
$$

where $\mathrm{H}(\phi)$ is the Heaviside function and D denotes the computational domain. In the numerical implementation, the level set function (LSF) is regularized to be a signed-distance function which does not only avoid too steep or too flat LSFs but also results in a smooth tensor field [58]. Moreover, the Courant-Friedrichs-Lewy (CFL) condition must be satisfied to improve the numerical stability while solving the H-J PDE [32].

\section{Numerical Implementation}

In numerical implementation, the unit strain field and periodic boundary condition can be equivalently replaced by symmetric and Dirichlet boundary conditions [65]. The 2D boundary value problem is solved using the commercial software COMSOL under plane stress assumption, where the elastic modulus tensor is provided in equation. Similarly, for the 3D cases the elastic tensor is provided in equation (10). This formulation gives the flexibility of creating microstructure with different behavior in different directions. In the current implementation, $C_{1111}$ and $C_{2222}$ have the same prescribed, and the expected Poison's ratio are $v_{12}=C_{1122} / C_{1111}$ for $2 \mathrm{D}$ and $v_{12}=C_{1122}^{H} /\left(C_{1111}^{H}+C_{1122}^{H}\right)$ for 3D. The flowchart of the optimization process is described Figure 6.

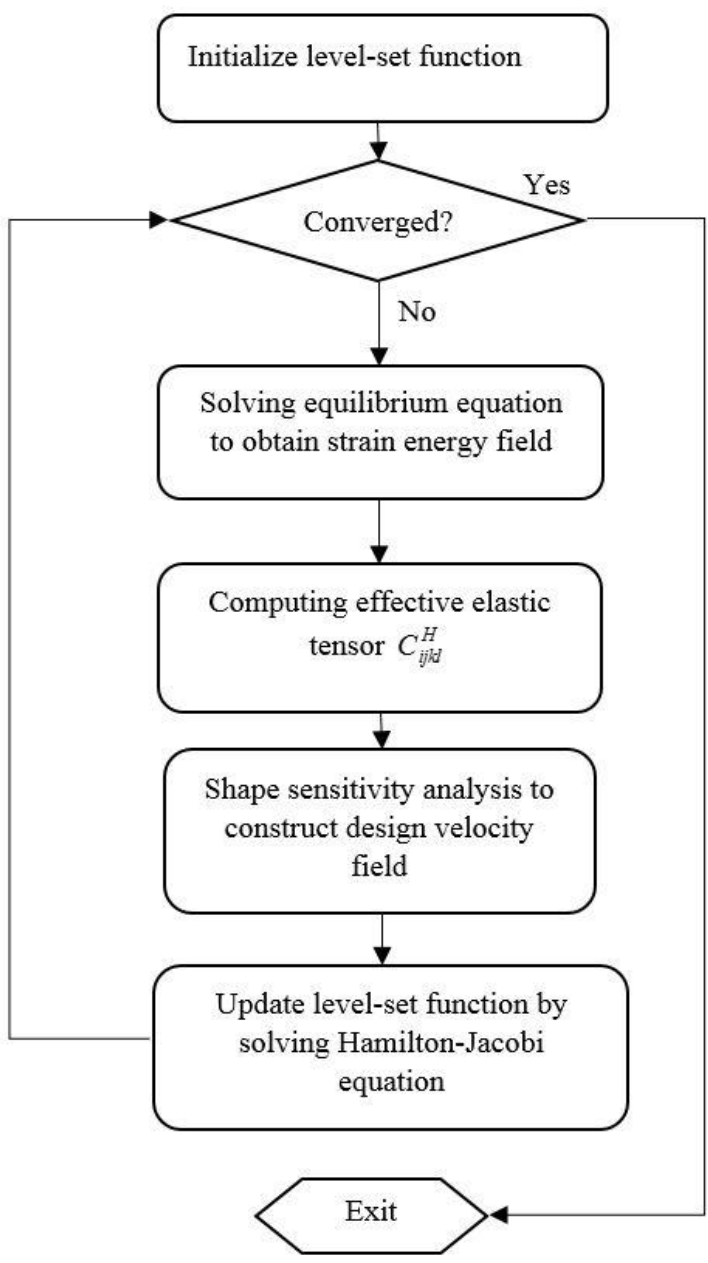

FIG. 6. Optimization flowchart 


\section{E. Shape Sensitivity Analysis}

To minimize the least square objective functional formulated in equation (15), we need to calculate the variation of the objective functional with respect to a variation of the boundary. In the current work, this process is done by using the material time derivative approach [66-68]. The Lagrange multiplier method is applied to couple the objective function with the governing equation. The design velocity field will be identified by taking the derivative of the Lagrange equation with respect to a pseudo-time $t$, which will be further integrated with the method of steepest descent to minimize the objective function.

The Lagrange equation is given by:

$$
L=J+\lambda g
$$

The derivative of the objective function with respect to the pseudo-time $t$ is obtained as:

$$
\frac{d J}{d t}=\sum_{i, j, k, l=1}^{d} w_{i j k l}\left(C_{i j k l}^{H}-C_{i j k l}^{*}\right) \frac{d C_{i j k l}^{H}}{d t}
$$

Since the effective properties are essentially the strain energy of the unit cell, the optimization problem of metamaterial design can be transformed to a series of mean compliance optimization problems under different Dirichlet boundary conditions. In each mean compliance optimization subproblem, the complete weak form of the governing equation with a weak imposition of Dirichlet boundary conditions can be rewritten as follows:

$$
g=\int_{\Omega} \varepsilon_{i j}^{T}(u) C_{i j k l} \varepsilon_{k l}(v) d \Omega-\beta \int_{\Gamma_{D}}\left(u-u_{0}\right) \cdot v d s .
$$

In equation (19), $\beta$ is the Lagrange multiplier and $u_{0}$ is the prescribed displacement on the boundary.

For the cases that $\mathrm{ij}=\mathrm{kl}(1111,2222$ and, 3333 for 3D), the effective elastic modulus can be expressed by the strain energy-based method as described in Section 2:

$$
C_{i j k l}^{H}=\int_{\Omega} \varepsilon_{i j}^{T}(u) C_{i j k l} \varepsilon_{k l}(u) d \Omega
$$

The derivative of the Lagrangian is expressed as:

$$
\begin{aligned}
\frac{D L}{D t}= & {\left[2 w_{i j k l}\left(C_{i j k l}^{H}-C_{i j k l}^{*}\right) \int_{\Omega} \varepsilon_{i j}^{T}\left(u^{\prime}\right) C_{i j k l} \varepsilon_{k l}(u) d \Omega+\int_{\Omega} \varepsilon_{i j}^{T}\left(u^{\prime}\right) C_{i j k l} \varepsilon_{k l}(v) d \Omega-\beta \int_{\Gamma_{D}} u^{\prime} \cdot v d s\right] . } \\
& +\left[w_{i j k l}\left(C_{i j k l}^{H}-C_{i j k l}^{*}\right) \int_{\Gamma} \varepsilon_{i j}^{T}(u) C_{i j k l} \varepsilon_{k l}(u) V_{n} d s+\int_{\Gamma} \varepsilon_{i j}^{T}(u) C_{i j k l} \varepsilon_{k l}(v) V_{n} d s\right]
\end{aligned}
$$

The first bracketed term is called the adjoint equation and the field variable $v$ is called the adjoint variable. The adjoint equation will vanish when the adjoint variable $v$ is set to be the following values:

$$
v=\left\{\begin{array}{ccc}
-2 w_{i j k l}\left(C_{i j k l}^{H}-C_{i j k l}^{*}\right) u, & \text { in } & \Omega \\
0 & \text { on } & \Gamma_{D}
\end{array}\right.
$$

By substituting $v$ into the convection terms in the second bracket, the shape sensitivity can be simplified as:

$$
\begin{aligned}
& w_{i j k l}\left(C_{i j k l}^{H}-C_{i j k l}^{*}\right) \int_{\Gamma} \varepsilon_{i j}^{T}(u) C_{i j k l} \varepsilon_{k l}(u) V_{n} d s+\int_{\Gamma} \varepsilon_{i j}^{T}(u) C_{i j k l} \varepsilon_{k l}(v) V_{n} d s \\
= & w_{i j k l}\left(C_{i j k l}^{H}-C_{i j k l}^{*}\right)\left[\int_{\Gamma} \varepsilon_{i j}^{T}(u) C_{i j k l} \varepsilon_{k l}(u) V_{n} d s+\int_{\Gamma} \varepsilon_{i j}^{T}(u) C_{i j k l} \varepsilon_{k l}(-2 u) V_{n} d s\right] \\
= & -w_{i j k l}\left(C_{i j k l}^{H}-C_{i j k l}^{*}\right) \int_{\Gamma} \varepsilon_{i j}^{T}(u) C_{i j k l} \varepsilon_{k l}(u) V_{n} d s
\end{aligned}
$$

With the steepest-descent method, the design velocity field can be constructed as: 


$$
V_{n}=w_{i j k l}\left(C_{i j k l}^{H}-C_{i j k l}^{*}\right) \varepsilon_{i j}^{T}(u) C_{i j k l} \varepsilon_{k l}(u)
$$

which is essentially the strain energy density of the unit cell under different loading cases.

In a similar way, the sensitivity analysis for the cases where $\mathrm{ij} \neq \mathrm{kl}$ (1122, and 1133 and 2233 for 3D) the velocity can be obtained as:

$$
V_{n}=\frac{1}{2} w_{1122}\left(C_{1122}^{H}-C_{1122}^{*}\right)\left[\varepsilon_{12}^{T}(u) C_{1122} \varepsilon_{12}(u)-\varepsilon_{11}^{T}(u) C_{1111} \varepsilon_{11}(u)-\varepsilon_{22}^{T}(u) C_{2222} \varepsilon_{22}(u)\right]
$$

By adding the mean curvature flow $\kappa$ for smoothing the boundaries and the contribution of the volume constraint to the sum of the velocities for each case, the whole design velocity field for the Lagrangian is defined as:

$$
V_{n}=\sum_{\text {case }=1}^{3 \text { or } 6} V_{n}^{\text {case }}+l_{1}\left(V-V^{*}\right)+l_{2} \kappa
$$

where $l_{1}$ and $l_{2}$ are the fixed Lagrangian multipliers for the volume and perimeter constraints respectively.

\section{NUMERICAL EXAMPLES}

In this section, the optimization procedure described above is applied to several benchmark examples. The following parameters are applied to all these examples, unless otherwise specified: the Young's modulus for the solid material is $\mathrm{E}=0.91 \mathrm{GPa}$ and for the dummy material $\mathrm{E}_{\mathrm{V}}=10^{-6} \mathrm{GPa}$; both with same Poisson's ratio equal to 0.3; the unit cell is discretized with 50-by-50 four-node quadrilateral elements.

This section begins with a demonstration of the optimization on a $2 \mathrm{D}$ design with specific prescribed elastic properties. Then, a parametric study is carried out with different volume constraints and Poisson's ratio targets, which aims to provide a complete overview of the potential designs. The effects of the initial designs as well as the Poisson's ratio targets are studied. Moreover, the optimization is applied in a multi-material problem with two material phases. At the end of this section, the procedure has also been extended to 3D problems, presenting unique single and multi-material designs of microstructures with negative Poisson's ratios.

\section{A. Designing 2D NPR Metamaterials with One Material}

The first example presents the topology optimization of a 2D unit cell with a target Poisson's ratio equal to $v=-0.4$. The evolution history of the geometry along with the resulted Poisson's ratio is provided with the plot of the objective function, as shown in Figure 7. The optimization starts with an initial design with multiple holes of void. The prescribed elastic properties targets are set as follows: $C_{1111}^{*}=0.2 \mathrm{GPa}, C_{1122}^{*}=-0.08 \mathrm{GPa}$ and $C_{2222}^{*}=0.2 \mathrm{GPa}$, which lead to a desired negative Poisson's ratio equal to -0.4. The weight factors of the optimization problem are set to be $w_{1111}=0.5, w_{2222}=0.5$, and $w_{1122}=5$ and the volume constraint is $60 \%$. Table III shows the optimal design of the unit cell, a 3-by-3 assembled periodic microstructure, and the corresponding effective properties. The iteration history, as well as selected intermediate results, are presented in Figure 7. The change of the elastic properties with the iteration number is plotted in Figure 8. During the optimization process, it can be seen that the least-square objective function is minimized gradually and finally reached zero in Figure 7. The elastic stiffness constants reach the targets after approximately 65 iterations as shown in Figure 8. The value of the Poisson's ratio for the optimal design becomes -0.4 as it was set to be. The result will be validated through experiment tests provided in Section 5 . 


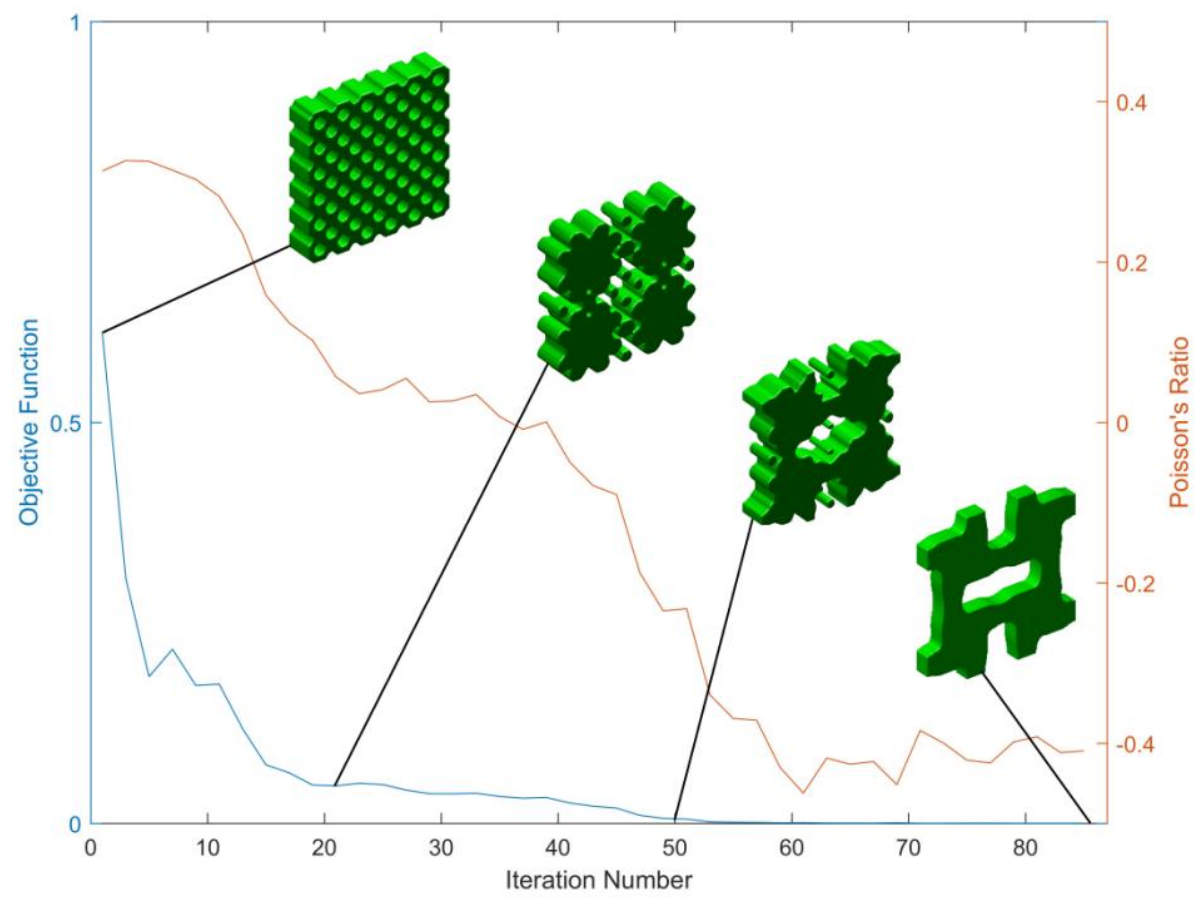

FIG. 7. The optimization history curve for the design with $v=-0.4$
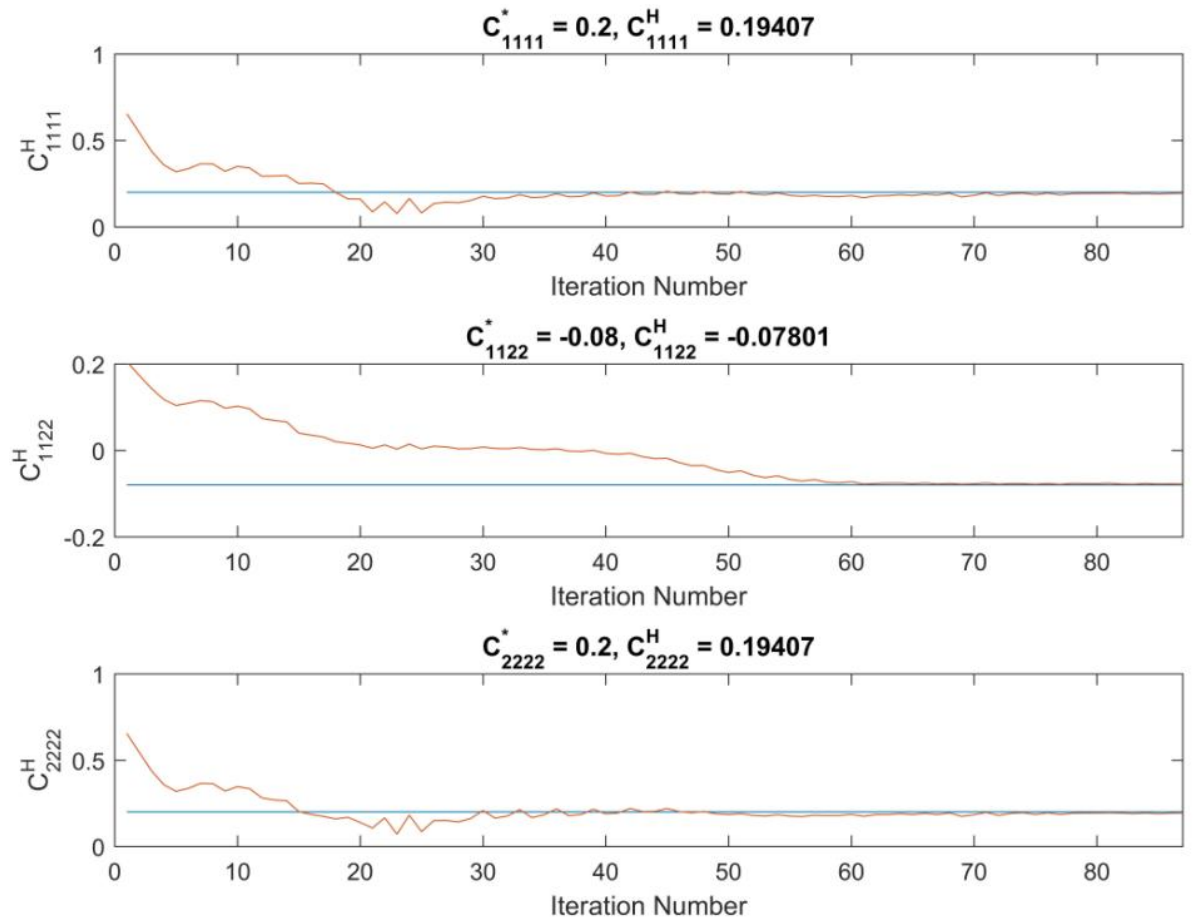

FIG. 8 . The history curves of elastic properties during optimization $(v=-0.4)$ 
TABLE III $v=-0.4$ : unit cell (left), $3 \times 3$ array (middle) and effective elastic properties (right) of the optimized design

Unit cell

Due to the limitations of steepest descent method and the fact that conventional level set methods do not provide nucleation mechanism for new holes, the design results strongly depend on the initial guess. A parametric study is carried out with same problem setting but different initial designs. In each of the following cases (Table IV), all the parameters were fixed, apart from the initial design, for better comparison. The results are shown in Table V, VI and VII.

TABLE IV Parameters for different cases

\begin{tabular}{|c|c|c|c|c|c|}
\hline Case & $C_{1111}^{*}(\mathrm{GPa})$ & $C_{2222}^{*}(\mathrm{GPa})$ & $C_{1122}^{*}(\mathrm{GPa})$ & $v$ & $V_{f}$ \\
\hline 1 & 0.2 & 0.2 & -0.04 & -0.2 & $50 \%$ \\
\hline 2 & 0.2 & 0.2 & -0.1 & -0.5 & $40 \%$ \\
\hline 3 & 0.2 & 0.2 & -0.1 & -0.5 & $50 \%$ \\
\hline
\end{tabular}

TABLE V Case 1: Optimal results from different initial designs with $v=-0.2$ and $V_{f}=50 \%$

Initial design Unit cell Elasticity Matrix [GPa]


TABLE VI Case 2: Optimal results from different initial designs with $v=-0.5$ and $V_{f}=40 \%$

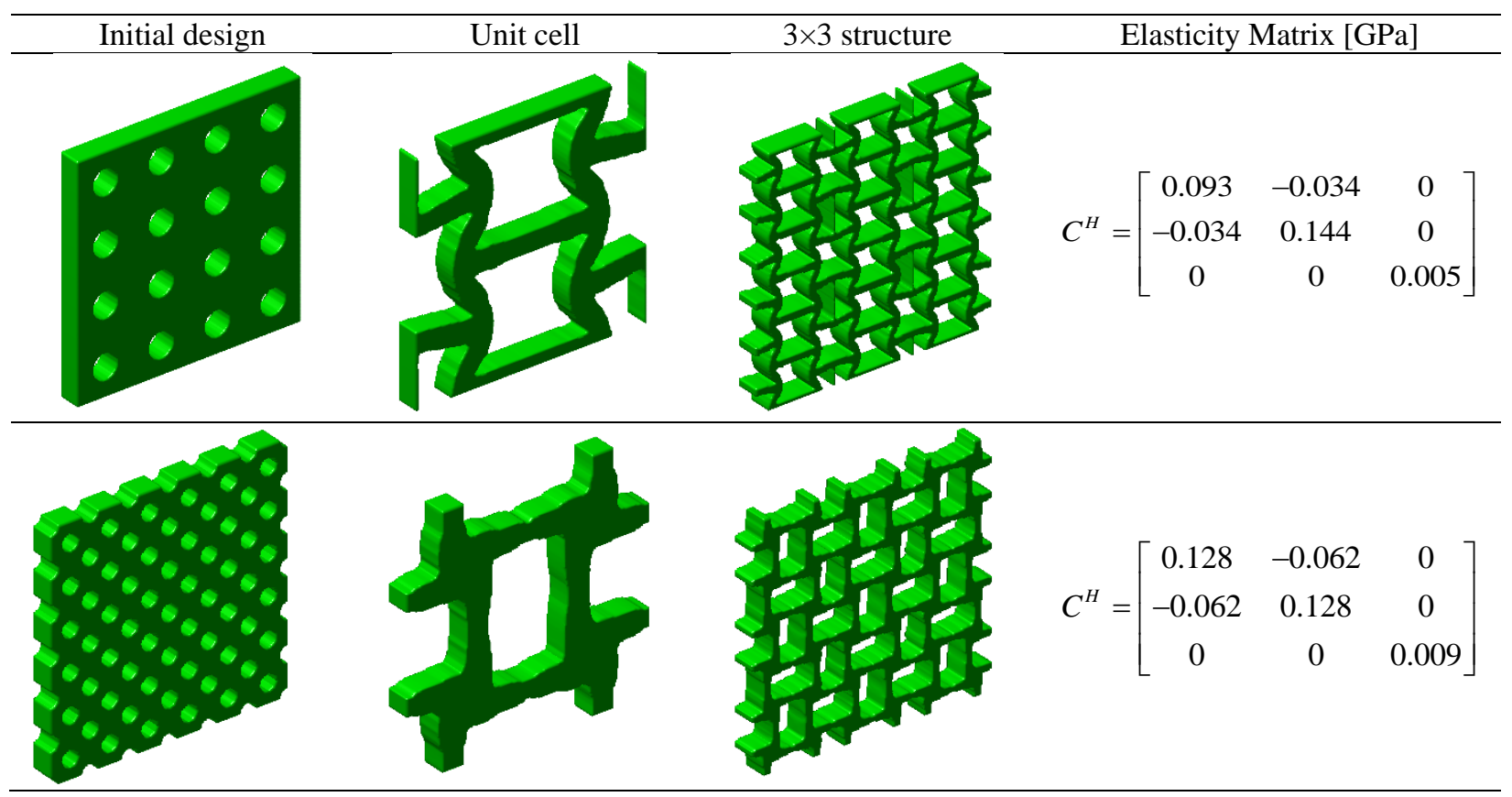

TABLE VII. Case 3: Optimal results from different initial designs with $v=-0.5$ and $V_{f}=50 \%$

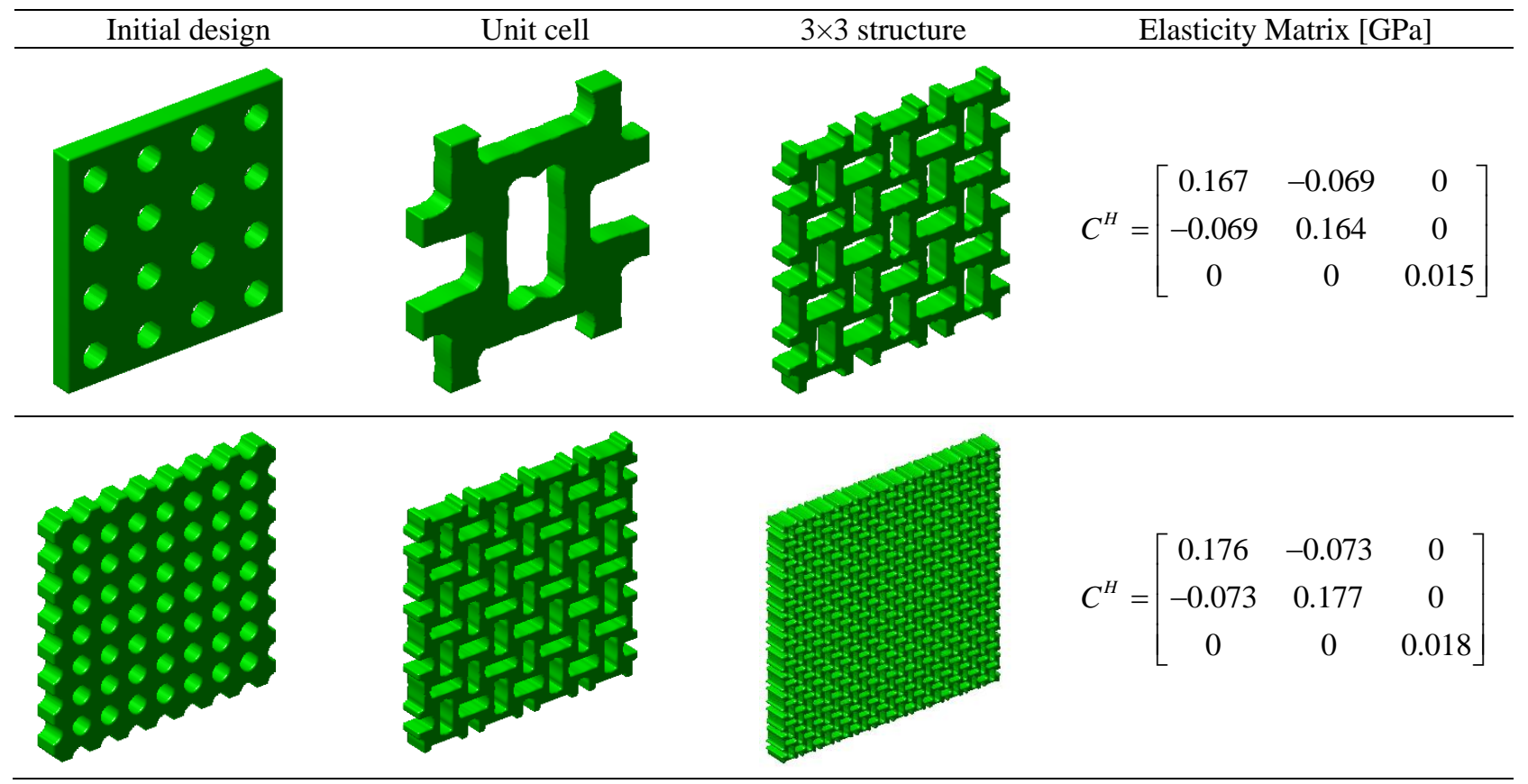

The resulted design will not always have the desired elastic properties. Since the material that is used for the current investigation has a specific Young's modulus of $0.91 \mathrm{GPa}$ and $v=0.3$, a design full of material will show $C_{1111}=1 G P a$. 
If target stiffness constant is set to be $C_{1111}^{*}=1 G P a$, the optimization will be able to offer such a behavior only if no volume constraint has been applied and the result would be full of material. But generally, it is not always possible to know a priori whether the elastic stiffness constant will be able to hit the specified targets in the least square objective function. In addition to that, an optimization with prescribed properties for the 3 elastic entries and a volume constraint usually result in a trade-off design without getting the desired effective properties. In case of not hitting the targets, one can get important feedback from the resulted geometry and change the problem settings accordingly. Having all the parameters along with the initial design constant, changing only the targets will drive to different designs and elastic tensors. In this section, two unit cells will be optimized to have the same Poisson's ratio -0.5 and same volume fraction but with different target for the elasticity tensors. The target elastic stiffness constants are set to be $C_{1111}^{*}=0.2 \mathrm{GPa}, C_{1122}^{*}=-0.1 \mathrm{GPa}$, and $C_{2222}^{*}=0.2 \mathrm{GPa}$ for unit cell 1 , and for unit cell 2, the targets would be $C_{1111}^{*}=0.1 \mathrm{GPa}, C_{1122}^{*}=-0.05 \mathrm{GPa}$, and $C_{2222}^{*}=0.1 \mathrm{GPa}$. The results are shown in Table VIII.

TABLE VIII. Optimal designs for the same Poisson's ratio -0.5 and volume fraction but different targets for the elasticity tensors

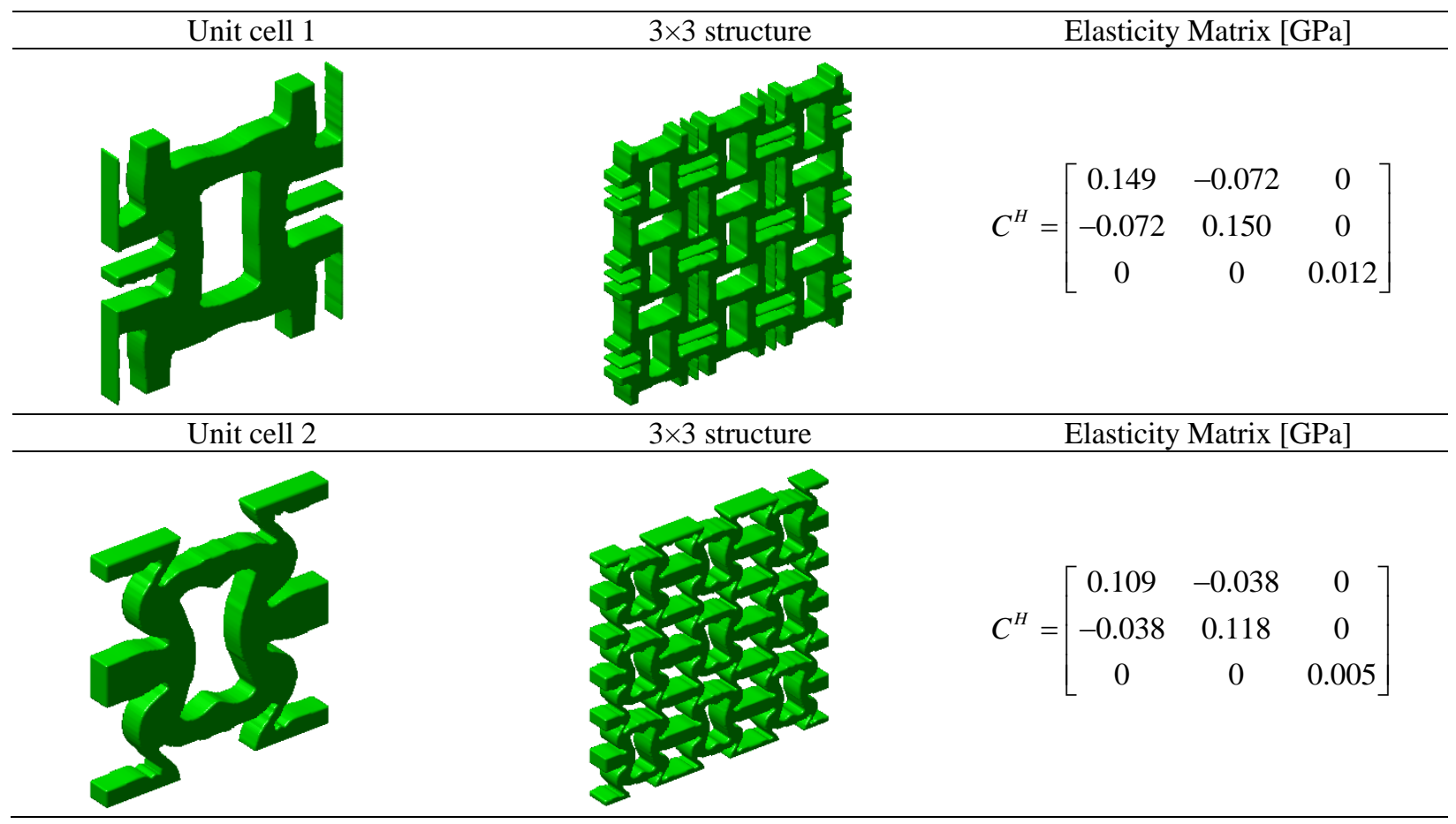

This section provides a series of examples with the same initial design, a 1-by-1 unit cell with 4 rows of 16 equally distributed holes, and the same targets for elastic stiffness constants $C_{1111}^{*}$ and $C_{2222}^{*}$. A parametric study can be helpful for the designer in terms of selecting the appropriate design or by giving important feedback for improving the optimization procedure. In this example, changing the desired Poisson's ratio $(v)$ and the volume constraint $\left(\mathrm{V}_{\mathrm{f}}\right)$ will drive the optimization to different results, giving an overall understanding of the possible patterns. For convenient comparison of the results, all other parameters were kept constant. By having $v$ as a variable, the prescribed elastic properties will be transformed into:

$$
C_{1111}^{*}=0.2 \mathrm{GPa}, \quad C_{1122}^{*}=-0.2 \nu \mathrm{GPa}, \quad C_{2222}^{*}=0.2 \mathrm{GPa}
$$




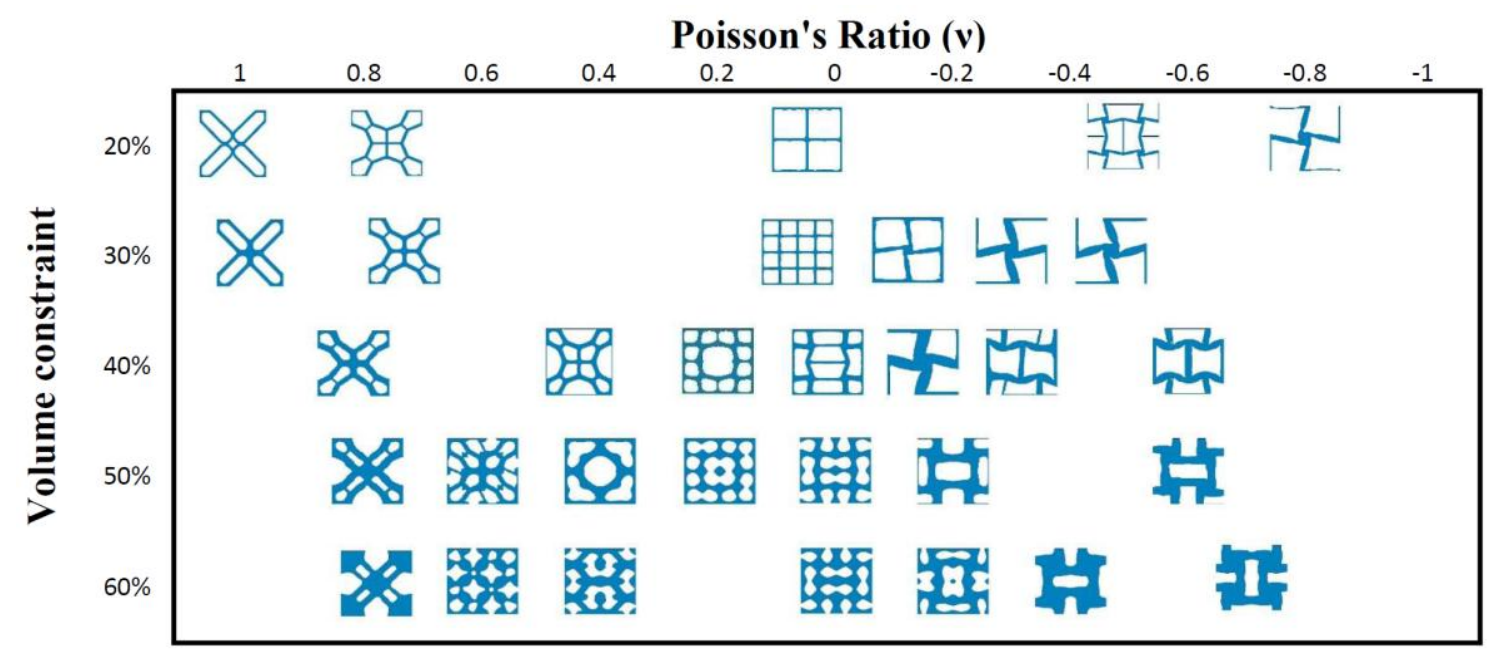

FIG. 9. Optimized auxetic metamaterials with various volume fractions and Poisson's ratios

Selected results of the parametric study are shown in Figure 9. It is worth noting that not all of the designs can reach the target of elastic tensors, or in other words, not all of the designs have the desired Poisson's ratio. Topology optimization is usually for continuum structures [18], while the design with Poisson's ratio equal to -1 may exist in a form of rigid body mechanisms. Besides the shape and topology of the unit cell, the effective Poisson's ratio also depends on how the internal structure deforms when the unit cell is loaded, and where and how the load is applied.

\section{B. Designing 2D NPR Metamaterials with Multiple Materials}

The reconcile level set method introduced in Section 3 is employed to optimize the unit cell. One additional material with a lower Young's Modulus is introduced. So, the problem is to simultaneously optimize the distribution of both the hard material with $\mathrm{E}_{H}=1 \mathrm{GPa}$ and the soft material with $\mathrm{E}_{S}=0.2 \mathrm{GPa}$. The void is represented with a dummy material with $\mathrm{E}_{V}=10^{-6} \mathrm{GPa}$. The volume fractions for the hard and soft material are $45 \%$ and $15 \%$, respectively. The targeted formulation is similar to the one in the single-material optimization with targets: $C_{1111}^{*}=0.2 G P a$, $C_{1122}^{*}=-0.1 G P a$, and $C_{2222}^{*}=0.2 G P a$ and target Poisson's ratio $v=-0.5$. The initial design is shown in Figure 10(a) and the final design is shown in Figure 10(b), where the green color refers to the hard material and the red color to the soft material. The 3-by-3 structures and elastic tensor are shown in Table IX. The iteration history, as well as selected intermediate result, are plotted in Figure 11. As can be seen in the final design, the soft material is distributed in a way which acts as de facto hinges undergoing large deformation to achieve negative Poisson's ratio without sacrificing the stiffness of the unit cell. In this example, the resulted Poisson's ratio reaches -0.38 , a value that will be verified in the next section.
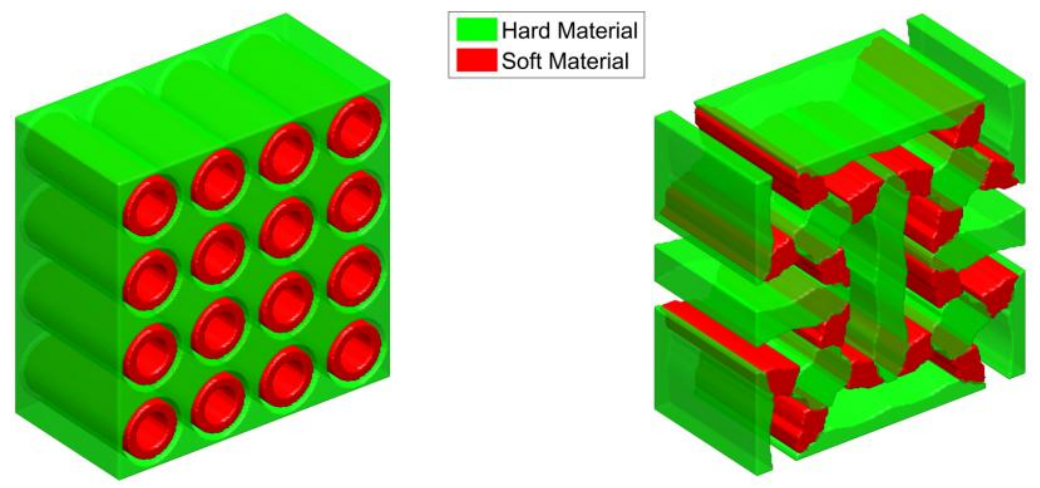

FIG. 10. The (a) Initial and (b) Final design of a 2D NPR metamaterial 
TABLE IX. The unit cell (left), 3x3 array (middle) and effective properties (right) for an optimal 2D multi-material NPR design

\begin{tabular}{ll}
\hline Unit cell & Elasticity Matrix [GPa] \\
\hline
\end{tabular}

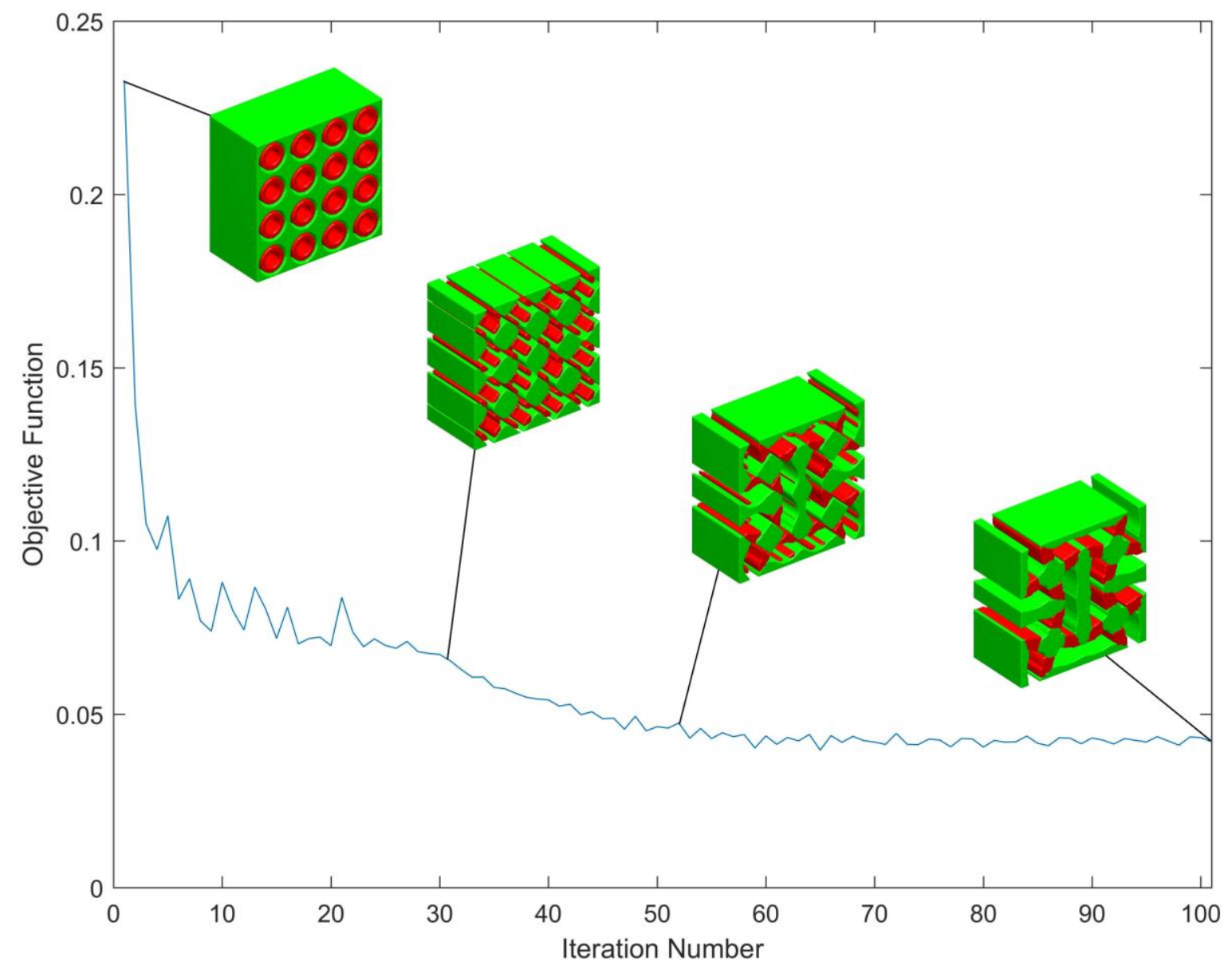

FIG. 11. The optimization history of a 2D multi-material NPR design

In another numerical test, the total volume fraction is fixed at $60 \%$, the different arrangement for volume fraction of hard material and soft material is investigated through following examples: (a) $40 \%$ for the hard material and $20 \%$ for the soft material; (b) $35 \%$ for the hard material and $25 \%$ for the soft material; (c) $30 \%$ for both hard and soft materials. The final designs of the unit cells are shown in Figure 12. 


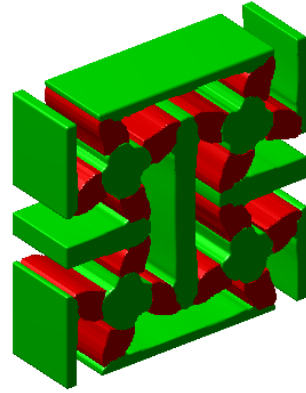

(a)

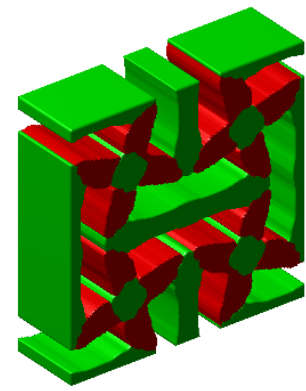

(b)

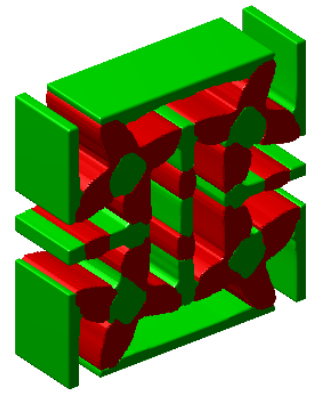

(c)

FIG. 12. Optimized 2D multi-material NPR designs with different volume fractions: (a) $40 \%$ for hard material and $20 \%$ for soft material; (b) $35 \%$ for hard material, and 25\% for soft material; (c) 30\% for both hard and soft materials. Green color for the hard material and red for the soft material.

\section{Designing 3D NPR Metamaterial with One Material}

The problem formulation has been extended in order to obtain a 3D unit cell with negative Poisson's ratio. The design domain consists of 40x40x40 elements and the initial design has uniformly distributed holes. The volume constraint is $25 \%$ and the weight factors follow the problem formulation of the $2 \mathrm{D}$ cases. In $3 \mathrm{D}$ cases, 6 elastic property targets have to be prescribed and, in the current optimization, are set as follows:

$C_{1111}^{*}=0.09 \mathrm{GPa}, C_{2222}^{*}=0.09 \mathrm{GPa}, C_{3333}^{*}=0.09 \mathrm{GPa}, C_{1122}^{*}=-0.03 \mathrm{GPa}, C_{1133}^{*}=-0.03 \mathrm{GPa}, C_{2233}^{*}=-0.03 \mathrm{GPa}$.

The above values imply a design with Poisson's ratio $v=-0.5$ in all three planes.

The initial design along with the obtained negative Poisson's ratio design are presented in Table X. The estimated Poisson's ratio value is -0.49 in all planes $\left(v_{12}=-0.4947, v_{13}=-0.4912, v_{23}=-0.4875\right)$ according to the elasticity matrix of the generated unit cell.

TABLE X. 3D single-material NPR unit cell structure: Initial (left), final (middle) designs of the unit cell and effective elastic properties (right) of the optimal design

Initial design

\section{Designing 3D NPR Metamaterials with Multiple Materials}

Similar to the 2D multi-material and 3D single-material investigation, an optimization has been formulated for a 3D multi-material NPR design. The design domain consists of 40x40x40 elements and the initial design has now 3 material phases: a box of hard material $\left(\mathrm{E}_{H}=1 \mathrm{GPa}\right)$ with $4 \times 4 \times 4$ uniformly distributed hollow spheres of soft 
material $\left(\mathrm{E}_{S}=0.2 \mathrm{GPa}\right)$; the third phase is the void inside the hollow spheres. The volume constraint is $12 \%$ for the hard material and $6 \%$ for the soft.

The initial design along with the obtained negative Poisson's ratio designs are presented in Tables XI and XIII. The full unit cell is obtained by mirroring the design in all three directions Tables XII and XIV.

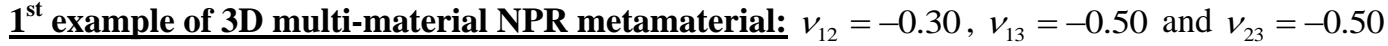

TABLE XI. $1^{\text {st }}$ example of 3D multi-material NPR design: Initial design (left), final design (middle) and constructed full unit cell (right)

Initial design Assembled unit cell

TABLE XII. Illustration of the $1^{\text {st }}$ example of 3D multi-material NPR design

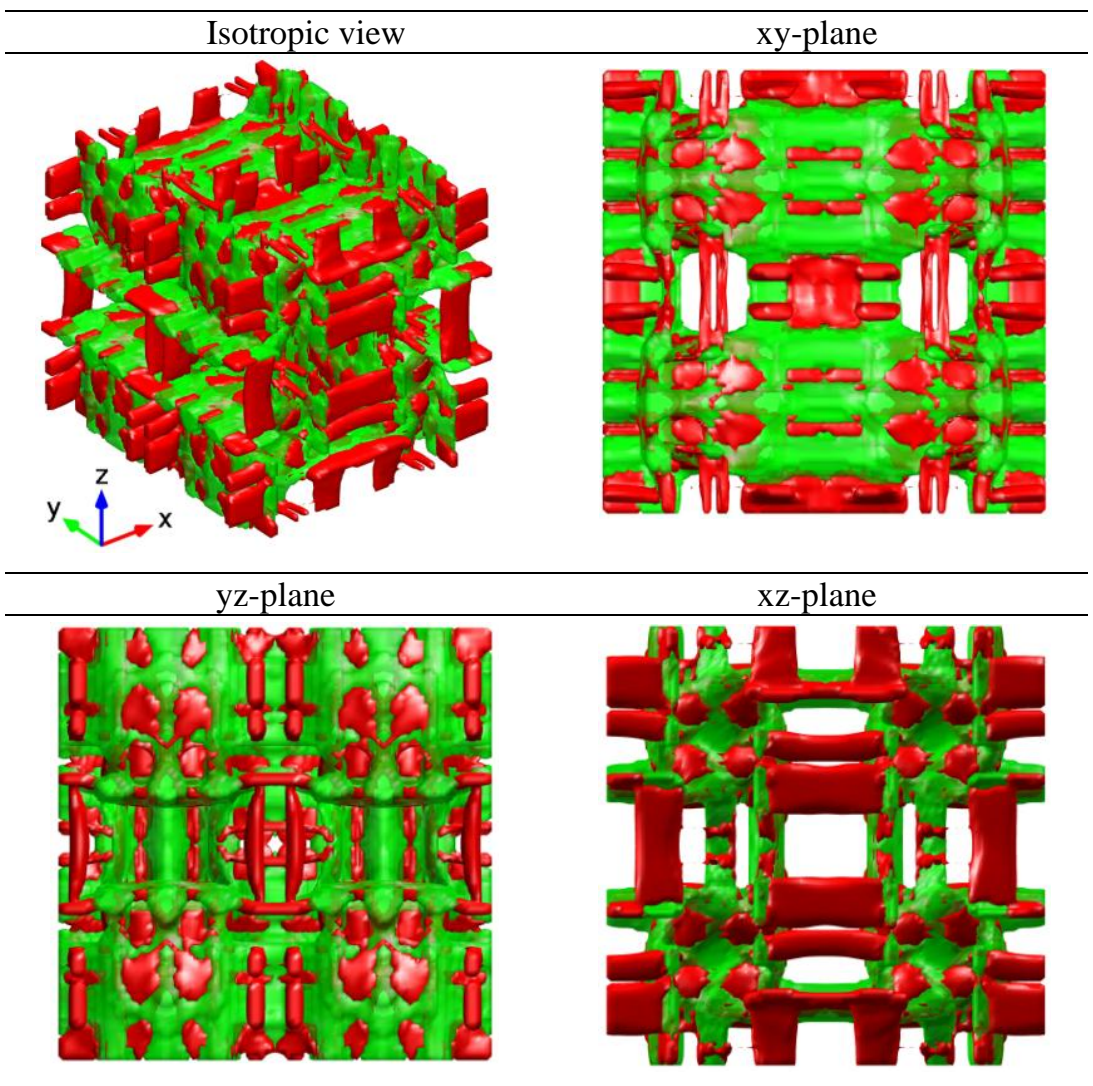


$\underline{2^{\text {nd }} \text { example of 3D multi-material NPR metamaterial: }} v_{12}=-0.90, v_{13}=-0.30$ and $v_{23}=-0.05$

TABLE XIII. $2^{\text {nd }}$ example of 3D multi-material NPR design: Initial design (left), final design (middle) and constructed full unit cell (right)

Initial design $1 / 8$ th of a unit cell Assembled unit cell

TABLE XIV. Illustration of the $2^{\text {nd }}$ example of 3D multi-material NPR design

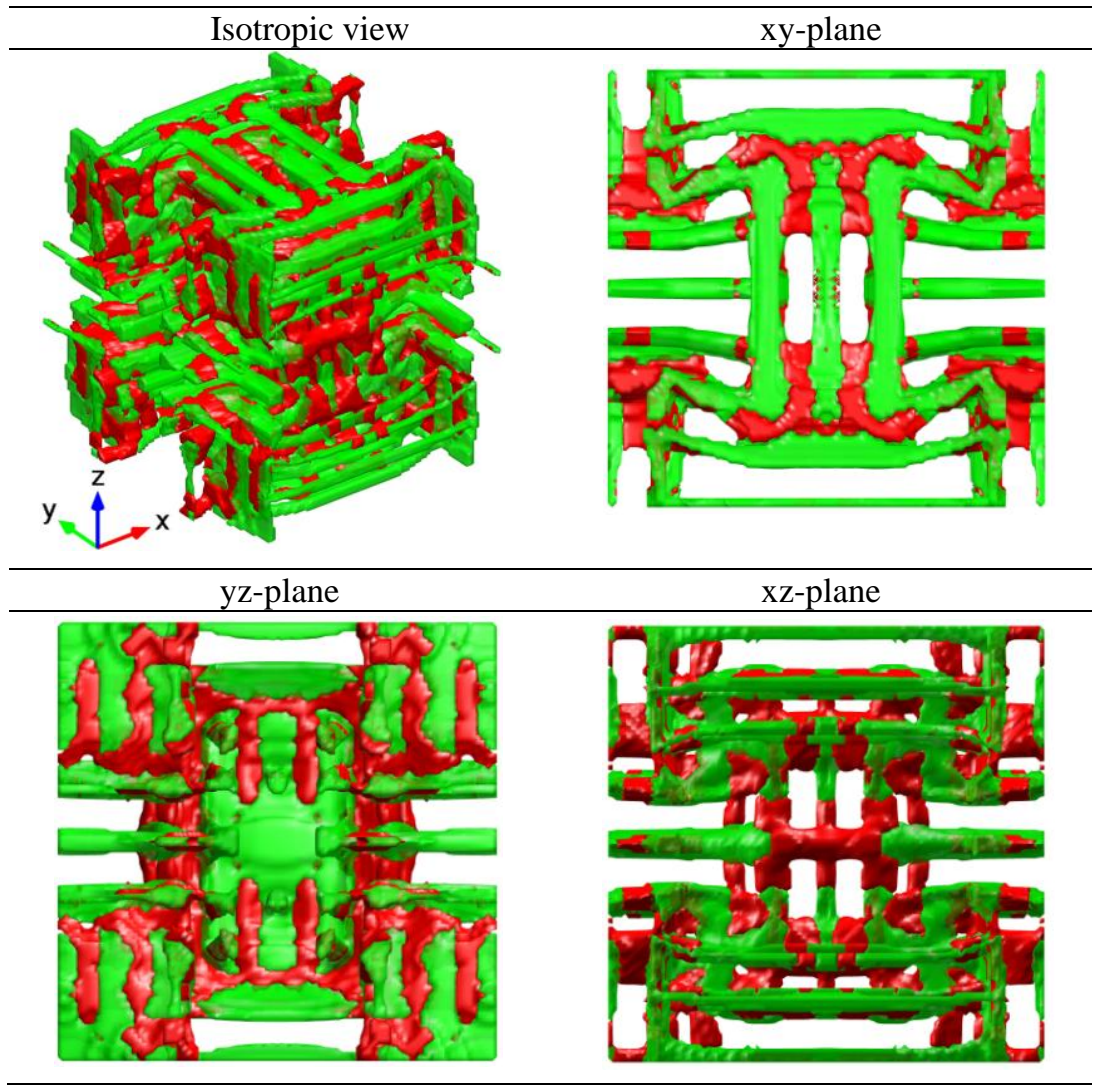




\section{NUMERICAL VERIFICATION and EXPERIMENTAL VALIDATION A. Verification and Validation of 2D Single-Material NPR Metamaterials}

The numerical verification was carried out using COMSOL Multiphysics for a $4 \times 4 \mathrm{~cm}$ structure with a volume fraction of 0.6 and targeted Poisson's ratio of -0.4. The boundary conditions are shown in Figure 13 (a), where two boundaries are supported and a displacement equal to $-0.4 \mathrm{~cm}$ is applied at the right end, which is equivalent to the effect of applying a uniform horizontal strain of $\varepsilon=-0.1$. In the simulation results, presented in Figure 13 (b), it can be clearly seen that the structure was shrinked in both $\mathrm{X}$ and $\mathrm{Y}$ directions and the displacement on upper boundary is around $-0.16 \mathrm{~cm}$ which gives a Poisson's ratio -0.4. The simulation results coroborate that the achieved metamaterial structure has negative Poisson's ratio $v=-0.4$.
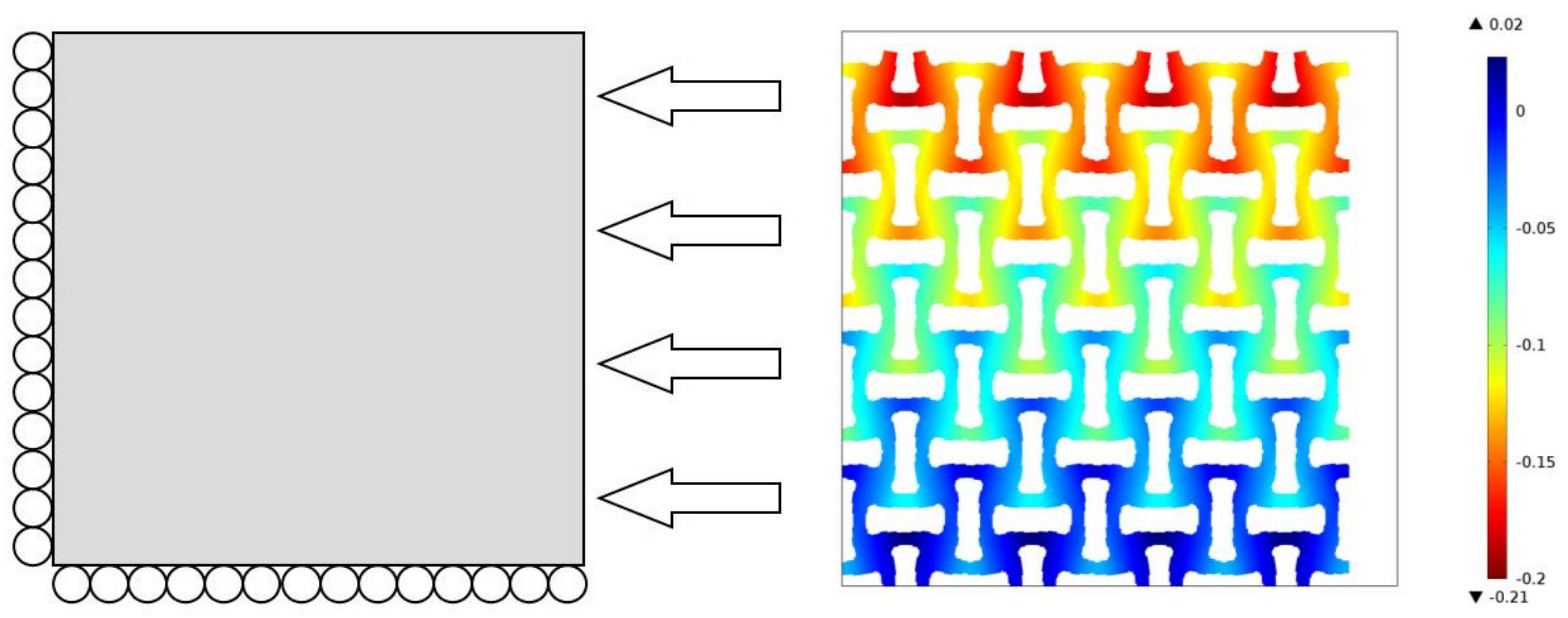

FIG. 13. Numerical verification of the auxetic behavior of the NPR metamaterial: (a) (left) Boundary conditions and (b) (right) simulation results.

The behavior of NPR metamaterials is further validated using experimental tests. Three prototype material structures are fabricated using a state-of-the-art multiple material 3D printer (Objet260 Connex, Stratasys Ltd) that allows the simultaneous printing of multi-materials in a single print. Tango Black, a rubber-like flexible material, is used for these structures. Compression tests are performed using a MTS mechanical testing machine (MTS Model43) with a $1 \mathrm{kN}$ load cell. The sample is held vertically between two metal bases. The upper and lower surface of the specimens are coated with liquid to reduce any frictional effects between the sample and the bases. The compression tests are performed by lowering the loader at a rate of $0.02 \mathrm{~mm} \mathrm{~s}^{-1}$. The deformation of the specimens is monitored using a high-resolution digital camera.

Three selected material structures are: specimen 1 with a volume fraction of 0.40 and targeted Poisson's ratio of 0.80 , specimen 2 with a volume fraction of 0.60 and targeted Poisson's ratio of -0.40 , and specimen 3 with a volume fraction of 0.60 and targeted Poisson's ratio of -0.80 . A representative sequence of images of specimens 1 and 2 during loading is presented in Figure 14 at different strains $\varepsilon=0,-0.05$, and -0.10 . Figure 14(a) clearly shows lateral expansion of specimen 1 during compression test, indicating a positive Poisson's ratio. On the contrary, Figure 14(b) shows lateral contraction during compression test, indicating a negative Poisson's ratio of specimen 3 . The mechanical response of three material structures are ploted in Figure 15(a), where stress-strain curves show slightly nonlinear behavior when the compression strain is larger than 0.09 , especially for specimen 3 . This is due to the local structure rotation and self-contacting in specimen 3 at large strain. The lateral and axial strains are obtained through image analysis at different strain levels. Figure 15(b) shows the lateral strain versus axial strain of all three speicimens. Therefore, the Poisson's ratio can be obtained as $0.82,-0.43$, and -0.77 for three specimens, respectively, which agrees very well with the simulated target values. These results indicate that the experimental tests verify the simulation results. 
(a)
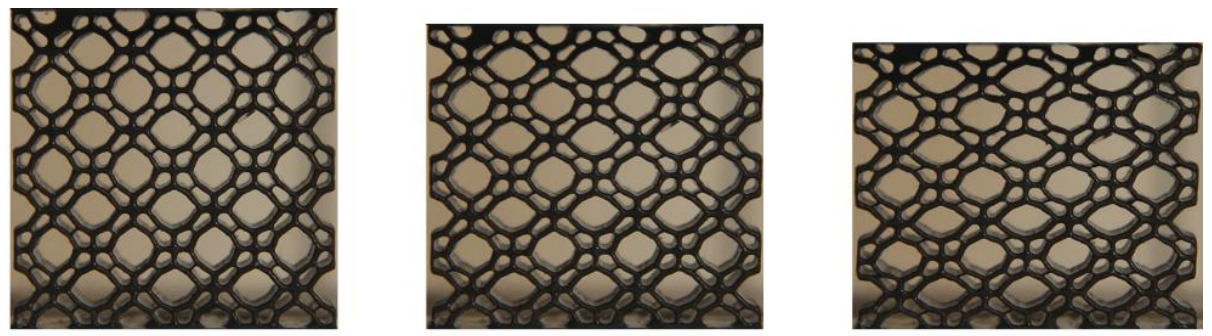

(b)
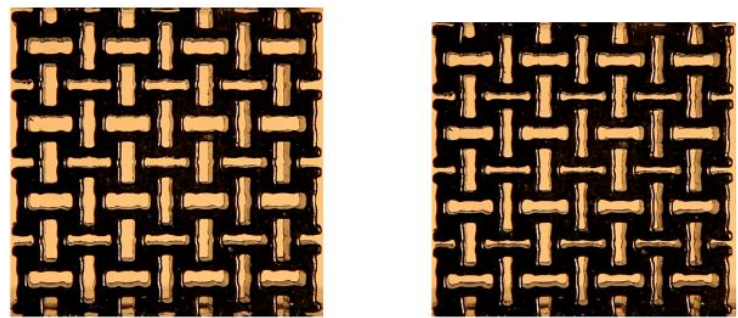

$\varepsilon=0.0$

$$
\varepsilon=-0.05
$$

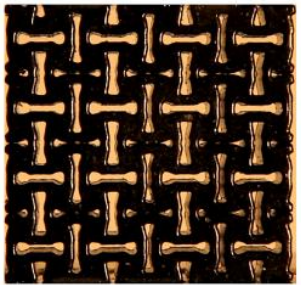

$\varepsilon=-0.10$

FIG. 14. Experimental images of the mechanical metamaterials at different levels of macroscopic strain: $0,-0.05$, and -0.10 . a) Specimen 1 with positive Poisson's ratio. b) Specimen 3 with negative Poisson's ratio.
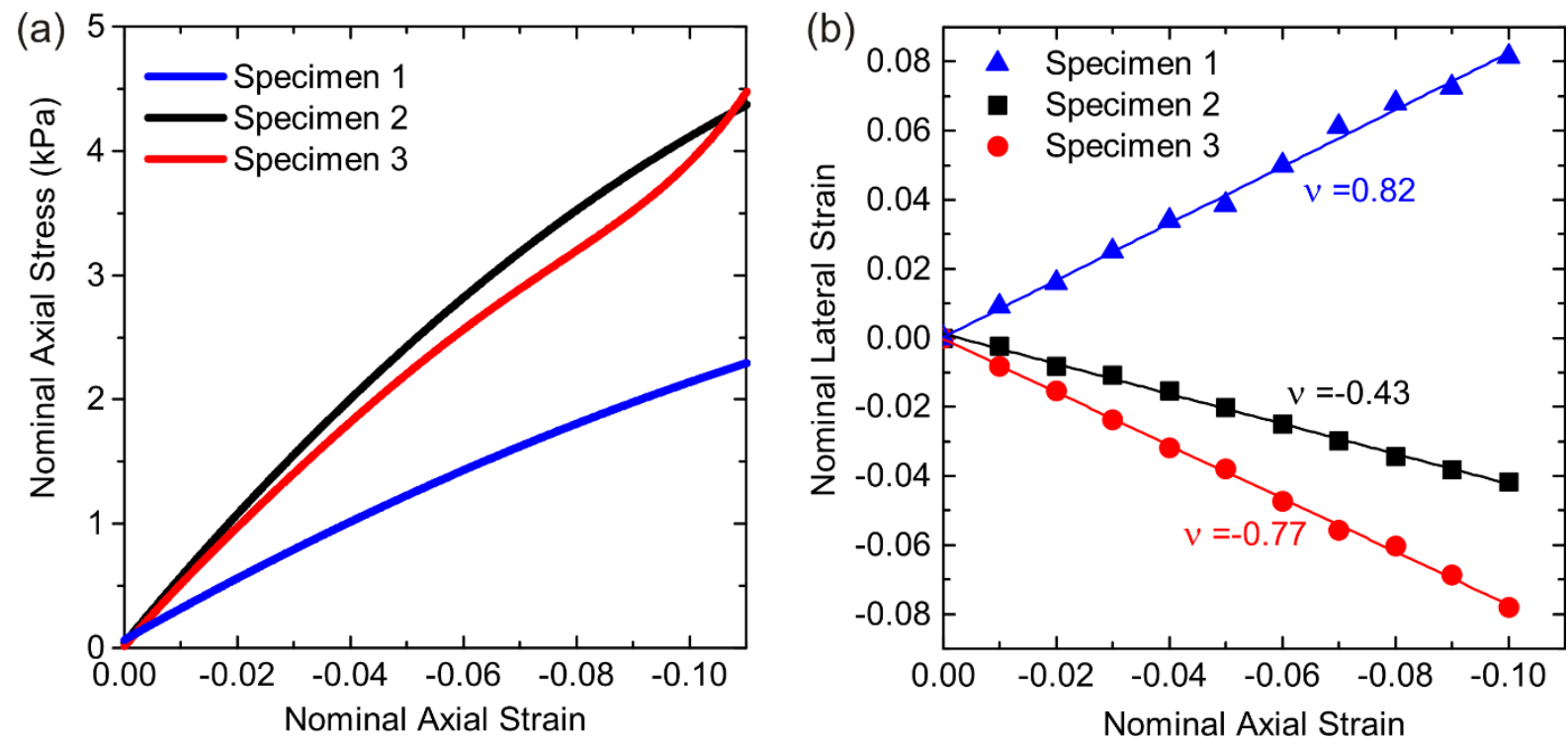

FIG. 15. Mechanical response of three mechanical metamaterials. a) Axial stress-strain curves of three specimens. b) Lateral strain versus axial strain 


\section{B. Verification and Validation of 2D Multi-material NPR Metamaterial}

Similarly, a numerical verification using COMSOL Multiphysics is performed for the multi-material design with total volume fraction of 0.6 (hard material: $45 \%$, soft material: $15 \%$ ) and resulted Poisson's ratio of -0.38 . The boundary conditions for the $4 \times 4 \mathrm{~cm}$ structure are the same as before, where two boundaries are supported and a displacement equal to $-0.4 \mathrm{~cm}$ is applied at the right end. In the simulation (Figure 16), the upper boundary is deformed by $-0.15 \mathrm{~cm}$ which is translated to a Poisson's ratio of -0.375 , which agrees with the estimated value.
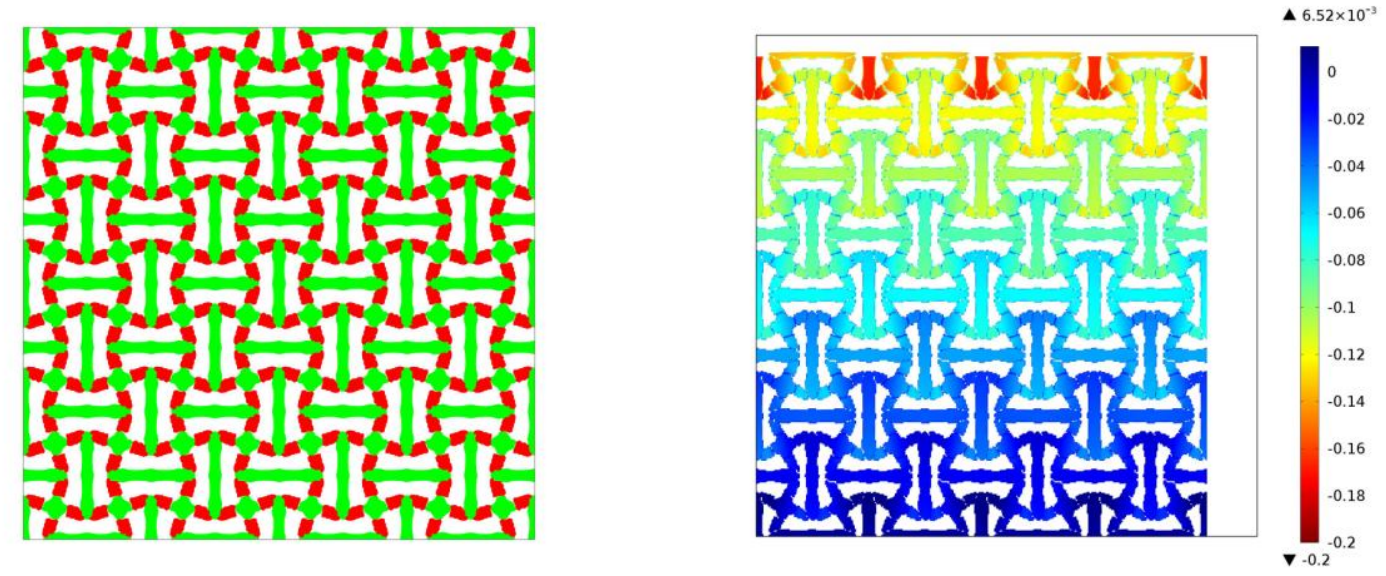

FIG. 16. Numerical verification of the auxetic behavior of the multi-material NPR metamaterial: (a) (left) Before and (b) (right) after applied displacement in $\mathrm{x}$ direction.

The above multi-material NPR structure is fabricated using the same 3D printer (Objet260 Connex, Stratasys Ltd). Vero white, a glassy polymer, is used as the hard material and Tango plus, a rubber-like material is used as the soft material. The compression test is performed using a MTS mechanical testing machine (MTS Model43) with a $1 \mathrm{kN}$ load cell. The experiment is conducted in a quasi-static regime with a constant rate of $0.02 \mathrm{mms}-1$.

A representative sequence of images of the specimen during loading is presented in Figure 17(a) at different strains $\varepsilon=0,-0.04$, and -0.08 . It is noted that the lateral contraction during compression test indicates a negative Poisson's ratio of the specimen. The mechanical response of the multi-material structure is ploted in Figture 17(b), where the stress-strain curve shows nonlinear behavior. This is due to the local structure rotation and self-contacting at large strain. To quantify the deformation taking place in the multi-material structure during the experiment, an image processing software (ImageJ) is used to determine the intersection points in the specimen. The deformation near the four edges of the specimen are strongly affected by boundary conditions. Therefore, the focus is on the behavior of the central part of the specimen as shown in Figure 17 (a). Finally, the lateral and axial strains are obtained at different strain levels. Figure 17(c) shows the lateral strain versus axial strain of both specimens, where the Poisson's ratio can be obtained as -0.36 verifying the simulation result. 
(a)
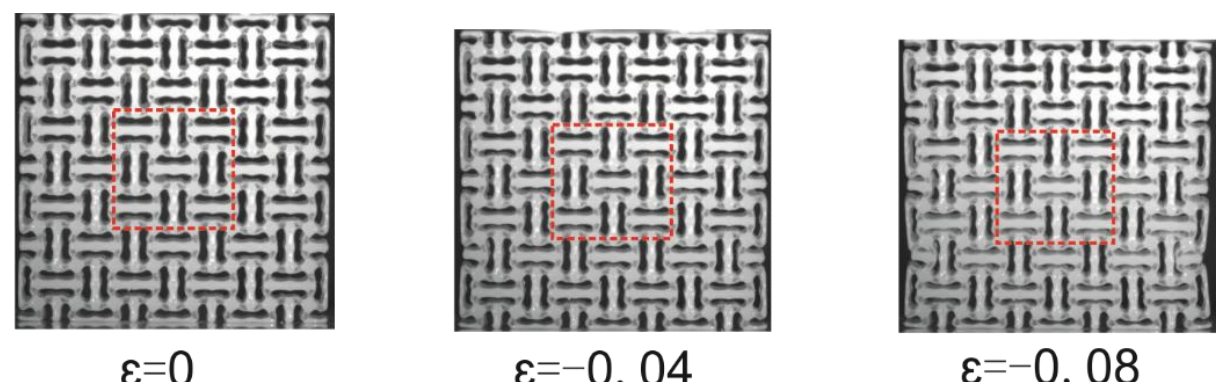

$\varepsilon=0$

$\varepsilon=-0.04$

$\varepsilon=-0.08$
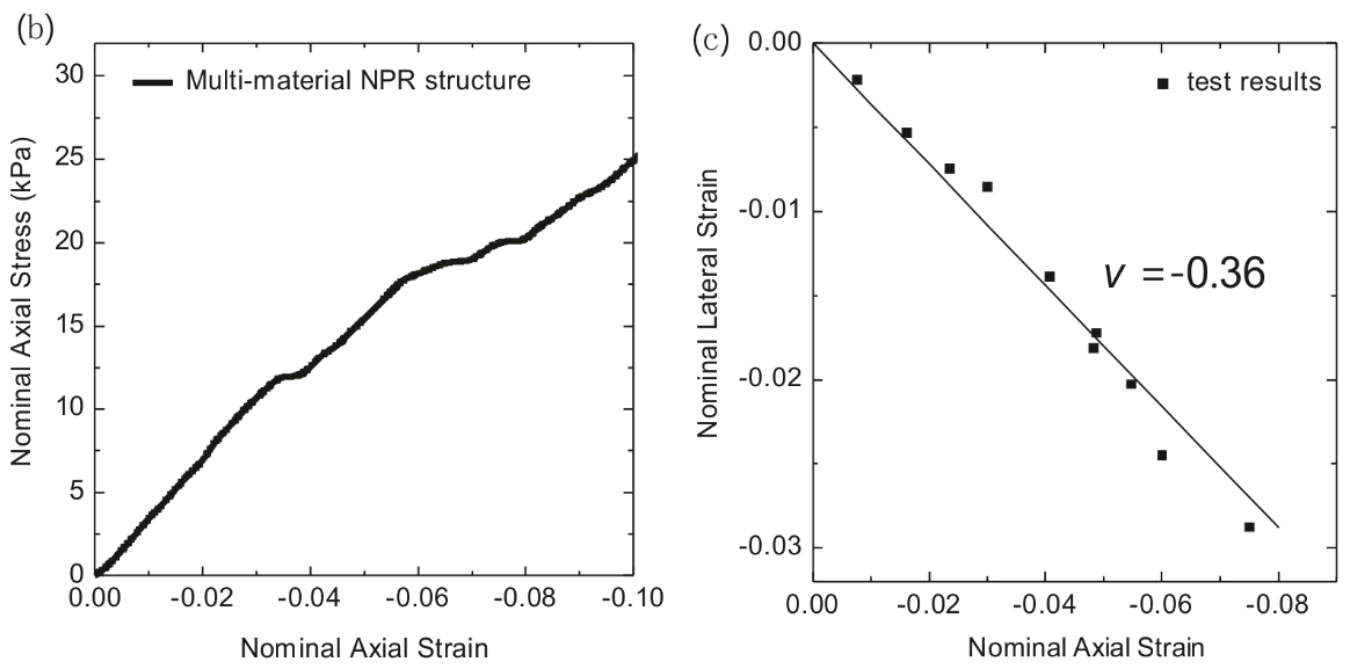

FIG. 17. Compression test for multi-material NPR structure. (a) Experimental images of the mechanical metamaterials at different levels of macroscopic strain: 0, -0.04, and -0.8. (b) Axial stress-strain curve. (c) Lateral strain versus axial strain. 


\section{Verification of 3D Single-Material NPR Metamaterial}

A $2 \times 2 \times 2 \mathrm{~cm}$ structure is built up from the 3D single-material unit cell for the verification model (Table XV). The mesh is refined, compared to the model used for the optimization process, increasing the accuracy of the result. The boundary conditions are the same as in the previous examples with $0.2 \mathrm{~cm}$ displacement on the right end leading to tension in $\mathrm{x}$ direction. The resulted displacement in the y direction is $0.1 \mathrm{~cm}$ and the Poisson's ratio is close to the estimation of -0.49 . The structure' deformation (xy-plane) is demonstrated in Figure 18. The structure has the same behavior in all 3 directions.

TABLE XV. 3D single material: Full unit cell assembled into a 2x2x2 structure.
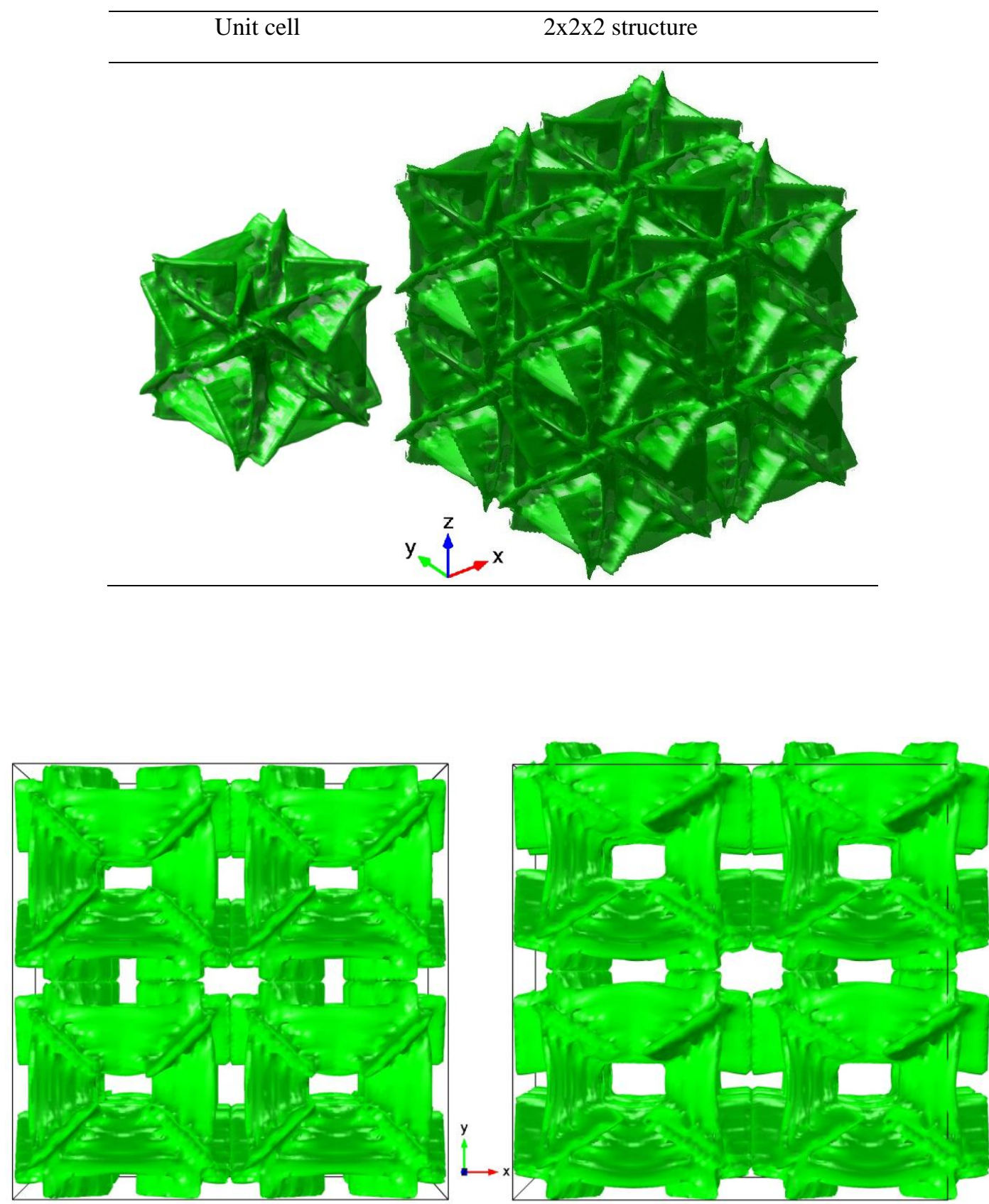

FIG. 18. Numerical verification of 3D NPR structure (xy-plane): (a) before (left) and (b) after (right) a displacement is applied in $\mathrm{x}$ direction. 


\section{Verification of 3D Multi-material NPR Metamaterials}

A $2 \times 2 \times 2 \mathrm{~cm} 3 \mathrm{D}$ multi-material structure, with refined mesh, is used for verification (TABLE XVI).

\section{$\underline{1^{\text {st }}}$ 3D multi-material NPR Metamaterial Example}

The boundary conditions are the same as in the previous examples with $0.2 \mathrm{~cm}$ displacement on the right end leading to tension in $\mathrm{x}$ direction (Figures 19-20). The resulted displacement in $\mathrm{y}$ direction is $0.06 \mathrm{~cm}$ and the Poisson's ratio verifies the estimation of $v_{12}=-0.30$. The displacement in $\mathrm{z}$ direction is $0.10 \mathrm{~cm}$ that agrees well with the estimated Poisson's ratio of $v_{13}=-0.50$. Furthermore, a second simulation is performed by applying a 0.2 $\mathrm{cm}$ displacement in y direction for obtaining the last Poisson's ratio of the structure (Figures 21). The structure deforms by $0.09 \mathrm{~cm}$ in $\mathrm{z}$ direction showing that $v_{23}=-0.45$.

TABLE XVI. The $1^{\text {st }}$ example of 3D multi-material NPR metamaterial: Full unit cell assembled into a $2 \times 2 \times 2 \mathrm{~cm}$ structure.

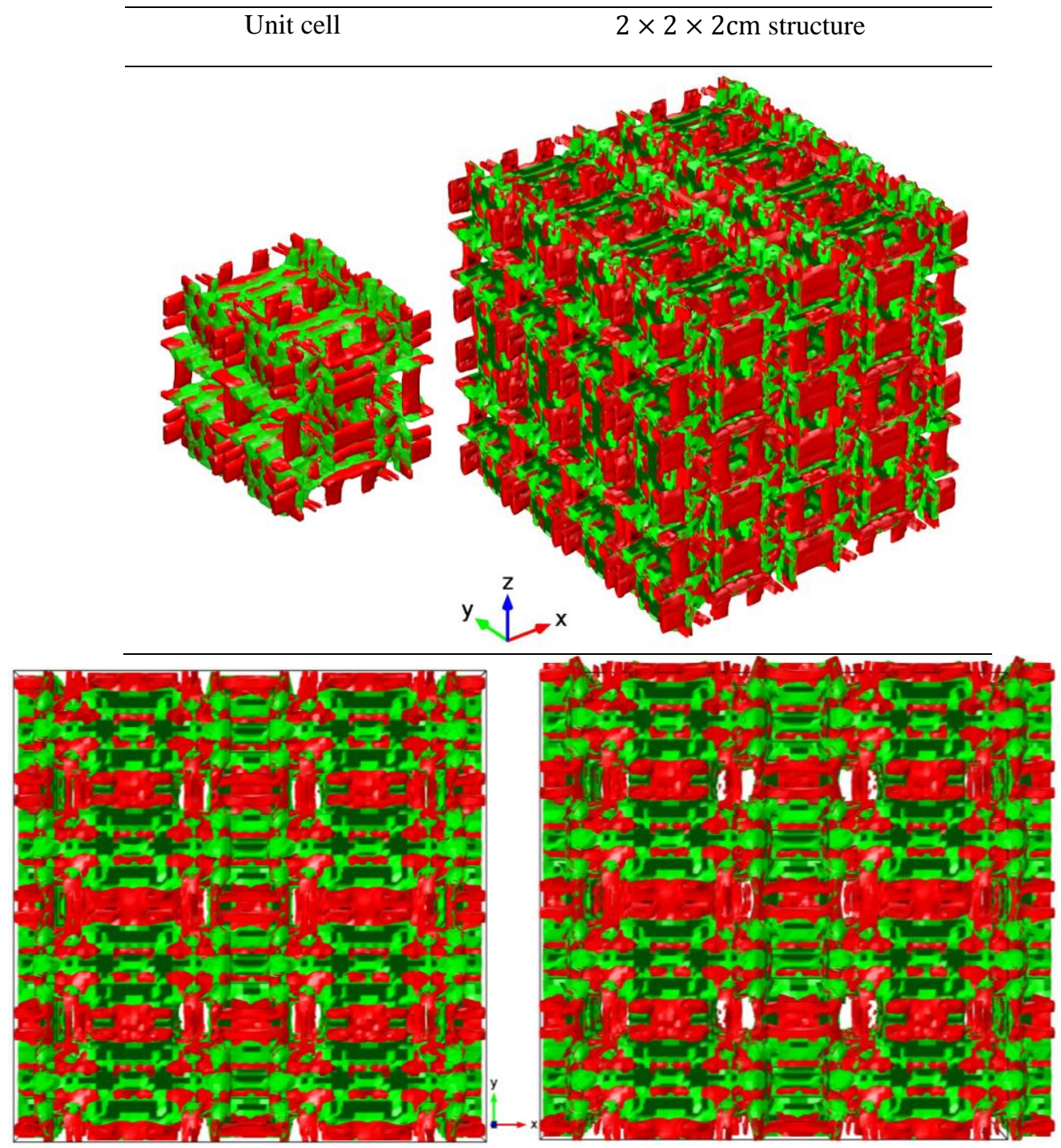

FIG. 19. Numerical verification of the $1^{\text {st }}$ example of 3D multi-material NPR structure (xy-plane): (a) before (left) and (b) after (right) a displacement is applied in $\mathrm{x}$ direction. 


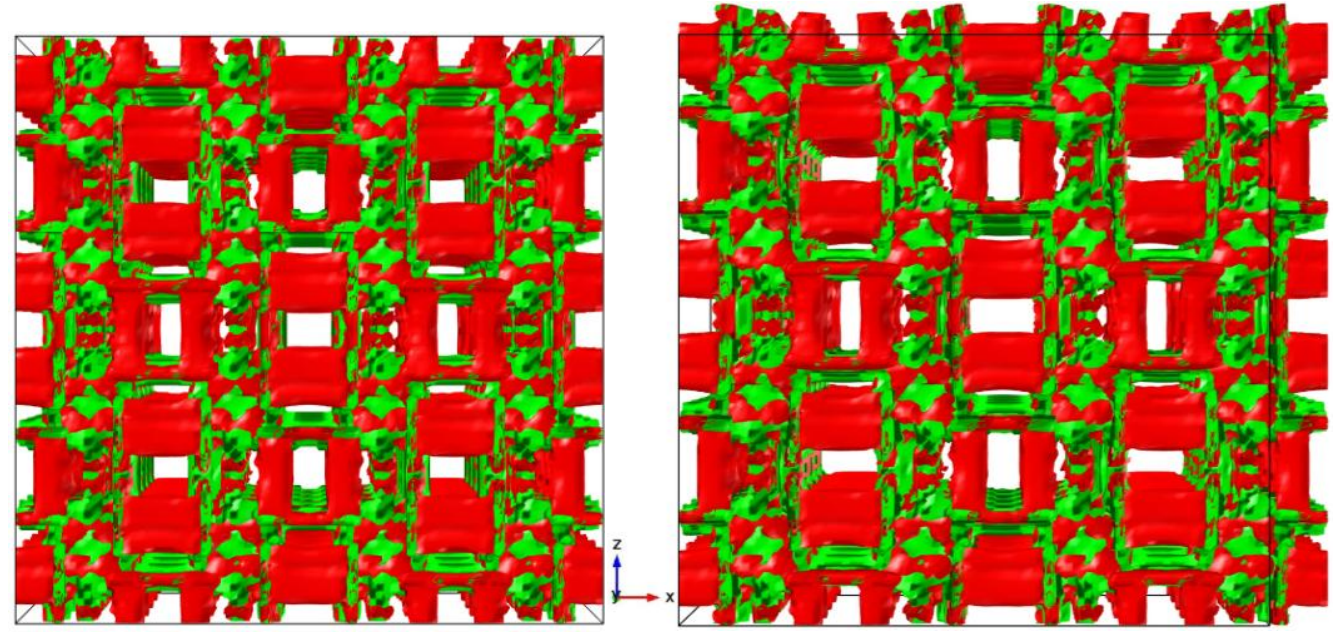

FIG. 20. Numerical verification of the $1^{\text {st }}$ example of 3D multi-material NPR structure (xz-plane): (a) before (left) and (b) after (right) a displacement is applied in $\mathrm{x}$ direction.

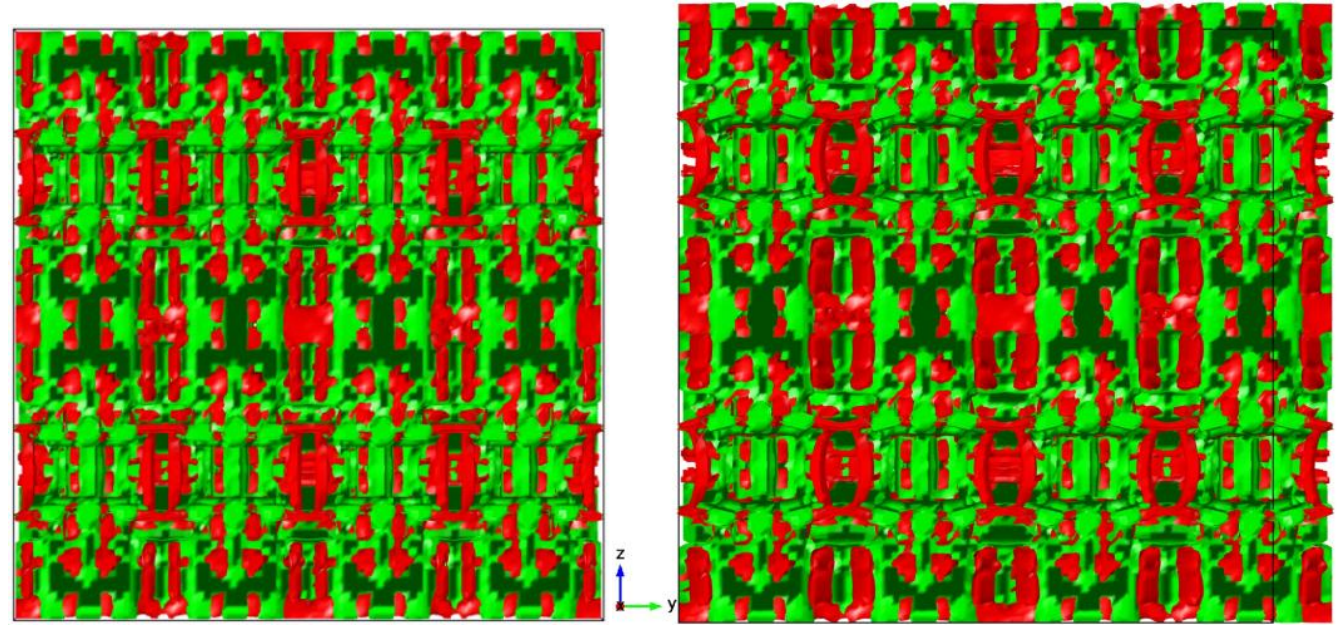

FIG. 21. Numerical verification of the $1^{\text {st }}$ example of 3D multi-material NPR structure (yz-plane): (a) before (left) and (b) after (right) a displacement is applied in y direction. 


\section{$\underline{2^{\text {nd }} 3 D}$ multi-material NPR Metamaterial Example}

Similarly, for the second 3D multi-material design (Table XVII), the Poisson's ratio values are: $v_{12}=-0.95$, $v_{13}=-0.3$ and $v_{23}=-0.05$ (Figures 22-24).

TABLE XVII. The $2^{\text {nd }}$ example of 3D multi-material NPR metamaterial: Full unit cell (left) assembled into a $2 \times 2 \times 2 \mathrm{~cm}$ structure.
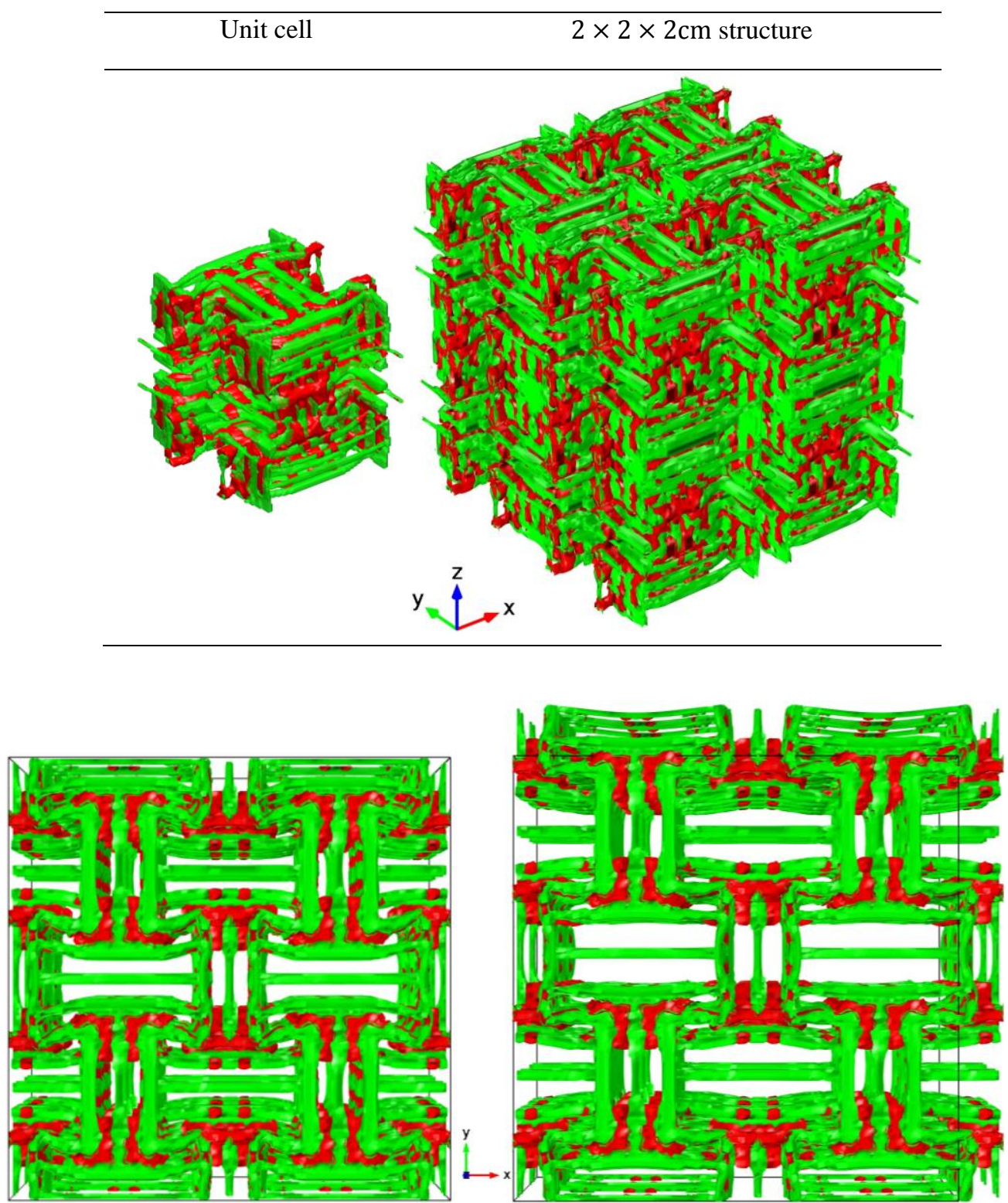

FIG. 22. Numerical verification of the $2^{\text {nd }} 3 \mathrm{D}$ multi-material NPR structure (xy-plane): (a) before (left) and (b) after (right) a displacement is applied in $\mathrm{x}$ direction. 


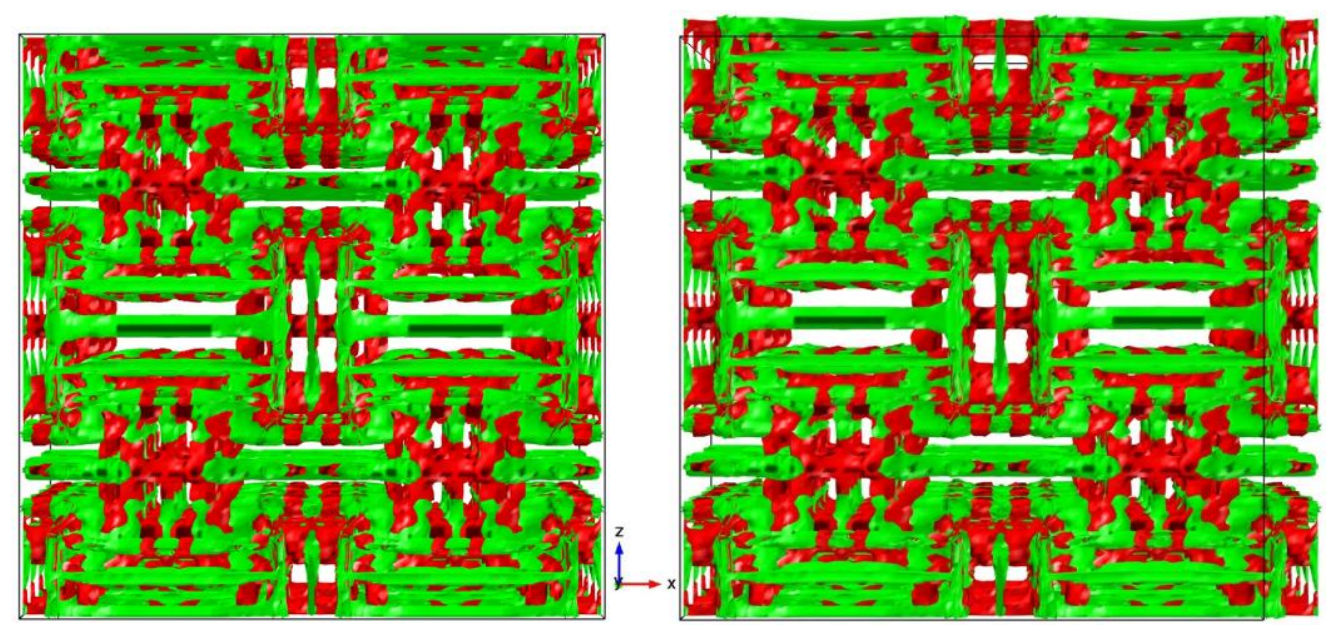

FIG. 23. Numerical verification of the $2^{\text {nd }}$ example of 3D multi-material NPR structure (xz-plane): (a) before (left) and (b) after (right) a displacement is applied in $\mathrm{x}$ direction.

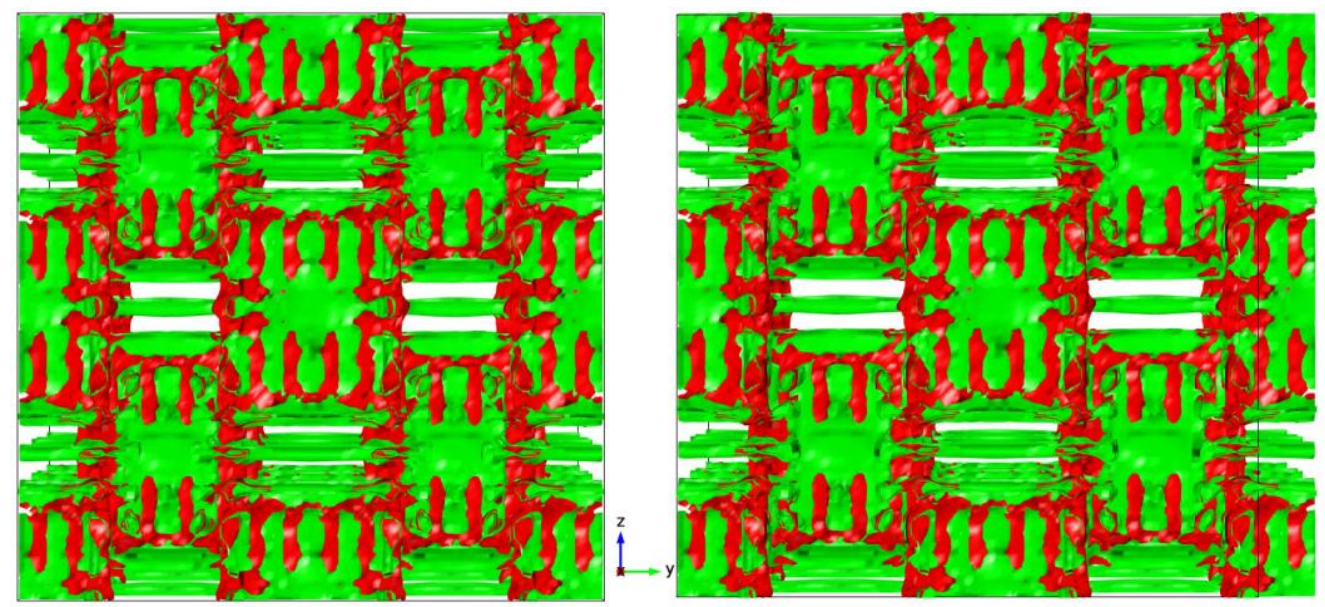

FIG. 24. Numerical verification of the $2^{\text {nd }}$ example of 3D multi-material NPR structure (yz-plane): (a) before (left) and (b) after (right) a displacement is applied in y direction.

\section{CONCLUSIONS}

This paper proposes a level set based method for topology optimization of both single and multiple-material NPR metamaterials. For multi-material topology optimization, the conventional level set method is advanced with a new approach exploiting the reconciled level set (RLS) method. The proposed method simplifies the conventional 'color' level set method by evolving each individual material with a single level set function and reconciling the resulted level set functions with the Merriman-Bence-Osher (MBO) operator. The NPR metamaterial design problem is formulated as a least square minimization problem using level set representation, which transforms the original design problem into a problem of finding the steady solution of the Hamilton-Jacobi equation. The shape sensitivity is rigorously derived based on the complete weak form of the governing equation with a weak imposition of Dirichlet boundary conditions. The $2 \mathrm{D}$ benchmark examples demonstrate the performance of the proposed method for metamaterial design. Both numerical simulations and physical experiments prove that the achieved design exhibit the desired auxetic behavior. The 2D single material designs follow the patterns of benchmark examples in existing literature. Novel designs are achieved for the 2D multi-material, and especially 3D single and multi-material matamaterials. The 3D design is enriching the currently limited available 3D designs with negative Poisson's ratio. The 3D multi-material design is innovative, and a new group of 3D multi-material microstructures with a different Poisson's ratio in each direction can be generated. One limitation in current approach is that it was hard to remove 
the supporting material when the 3D designs are fabricated through additive manufacturing. In our future work, we will take into account such additive manufacturing constraints in the topology design process.

\section{Acknowledgements}

Thanks go to the anonymous reviewers for their helpful and constructive comments. The authors acknowledge the support from the National Science Foundation (CMMI1462270), the Region 2 University Transportation Research Center (UTRC) and the start-up funds from the State University of New York at Stony Brook. 


\section{References}

[1] Q. Liu, Literature review: materials with negative Poisson's ratios and potential applications to aerospace and defence, in, DTIC Document, 2006.

[2] J. Choi, R. Lakes, Fracture toughness of re-entrant foam materials with a negative Poisson's ratio: experiment and analysis, International Journal of fracture, 80 (1996) 73-83.

[3] J. Donoghue, K. Alderson, K. Evans, The fracture toughness of composite laminates with a negative Poisson's ratio, physica status solidi (b), 246 (2009) 2011-2017.

[4] C. Chen, R. Lakes, Micromechanical analysis of dynamic behavior of conventional and negative Poisson's ratio foams, Journal of Engineering Materials and Technology, 118 (1996) 285-288.

[5] R. Lakes, Foam structures with a negative Poisson's ratio, Science, 235 (1987) 1038-1040.

[6] A. Alderson, K. Alderson, Auxetic materials, Proceedings of the Institution of Mechanical Engineers, Part G: Journal of Aerospace Engineering, 221 (2007) 565-575.

[7] K.E. Evans, A. Alderson, Auxetic Materials: Functional Materials and Structures from Lateral Thinking!, Advanced Materials, 12 (2000) 617-628.

[8] R. Lakes, Advances in negative Poisson's ratio materials, Advanced Materials, 5 (1993) 293-296.

[9] K. Bertoldi, P.M. Reis, S. Willshaw, T. Mullin, Negative Poisson's ratio behavior induced by an elastic instability, Advanced Materials, 22 (2010) 361-366.

[10] S. Babaee, J. Shim, J.C. Weaver, E.R. Chen, N. Patel, K. Bertoldi, 3D Soft metamaterials with negative Poisson's ratio, Advanced Materials, 25 (2013) 5044-5049.

[11] L. Wang, M.C. Boyce, Bioinspired Structural Material Exhibiting Post-Yield Lateral Expansion and Volumetric Energy Dissipation During Tension, Advanced Functional Materials, 20 (2010) 3025-3030.

[12] F. Song, J. Zhou, X. Xu, Y. Xu, Y. Bai, Effect of a negative Poisson ratio in the tension of ceramics, Physical review letters, 100 (2008) 245502.

[13] J. Ciambella, A. Bezazi, G. Saccomandi, F. Scarpa, Nonlinear elasticity of auxetic open cell foams modeled as continuum solids, Journal of Applied Physics, 117 (2015) 184902.

[14] V. Aleshin, I. Raevski, Negative Poisson's ratio and piezoelectric anisotropy of tetragonal ferroelectric single crystals, Journal of Applied Physics, 112 (2012) 114101.

[15] A. Kolpakov, Determination of the average characteristics of elastic frameworks, Journal of Applied Mathematics and Mechanics, 49 (1985) 739-745.

[16] R. Almgren, An isotropic three-dimensional structure with Poisson's ratio =-1, Journal of elasticity, 15 (1985) 427-430.

[17] P. Theocaris, G. Stavroulakis, P. Panagiotopoulos, Negative Poisson's ratios in composites with star-shaped inclusions: a numerical homogenization approach, Archive of Applied Mechanics, 67 (1997) 274-286.

[18] M.P. Bendsoe, O. Sigmund, Topology optimization: theory, methods and applications, Springer, 2003.

[19] H.A. Eschenauer, N. Olhoff, Topology optimization of continuum structures: A review*, Applied Mechanics Reviews, 54 (2001) 331-390.

[20] G. Rozvany, Aims, scope, methods, history and unified terminology of computer-aided topology optimization in structural mechanics, Structural and Multidisciplinary Optimization, 21 (2001) 90-108.

[21] O. Sigmund, K. Maute, Topology optimization approaches, Structural and Multidisciplinary Optimization, 48 (2013) 1031-1055.

[22] O. Sigmund, Materials with prescribed constitutive parameters: An inverse homogenization problem, International Journal of Solids and Structures, 31 (1994) 2313-2329.

[23] O. Sigmund, Tailoring materials with prescribed elastic properties, Mechanics of Materials, 20 (1995) 351-368.

[24] M.Y. Wang, X. Wang, A level-set based variational method for design and optimization of heterogeneous objects, Computer-Aided Design, 37 (2005) 321-337.

[25] G. Allaire, F. De Gournay, F. Jouve, A. Toader, Structural optimization using topological and shape sensitivity via a level set method, Control and cybernetics, 34 (2005) 59.

[26] W. Zhang, G. Dai, F. Wang, S. Sun, H. Bassir, Using strain energy-based prediction of effective elastic properties in topology optimization of material microstructures, Acta Mechanica Sinica, 23 (2007) 77-89.

[27] B. Xu, F. Arias, S.T. Brittain, X.-M. Zhao, B. Grzybowski, S. Torquato, G.M. Whitesides, Making negative Poisson's ratio microstructures by soft lithography, Advanced materials, 11 (1999) 1186.

[28] J. Schwerdtfeger, F. Wein, G. Leugering, R. Singer, C. Körner, M. Stingl, F. Schury, Design of auxetic structures via mathematical optimization, Advanced materials, 23 (2011) 2650-2654.

[29] E. Andreassen, B.S. Lazarov, O. Sigmund, Design of manufacturable 3D extremal elastic microstructure, Mechanics of Materials, 69 (2014) 1-10. 
[30] S. Shan, S.H. Kang, Z. Zhao, L. Fang, K. Bertoldi, Design of planar isotropic negative Poisson's ratio structures, Extreme Mechanics Letters, 4 (2015) 96-102.

[31] A. Clausen, F. Wang, J.S. Jensen, O. Sigmund, J.A. Lewis, Topology Optimized Architectures with Programmable Poisson's Ratio over Large Deformations, Advanced Materials, 27 (2015) 5523-5527.

[32] S. Osher, J.A. Sethian, Fronts propagating with curvature-dependent speed: Algorithms based on HamiltonJacobi formulations, Journal of Computational Physics, 79 (1988) 12-49.

[33] M.Y. Wang, X. Wang, D. Guo, A level set method for structural topology optimization, Computer methods in applied mechanics and engineering, 192 (2003) 227-246.

[34] G. Allaire, F. Jouve, A.-M. Toader, Structural optimization using sensitivity analysis and a level-set method, Journal of computational physics, 194 (2004) 363-393.

[35] D. Cioranescu, J.S.J. Paulin, Homogenization in open sets with holes, Journal of mathematical analysis and applications, 71 (1979) 590-607.

[36] E. Sanchez-Palencia, A. Zaoui, Homogenization techniques for composite media, in: Homogenization Techniques for Composite Media, 1987.

[37] G. Allaire, Homogenization and Two-Scale Convergence, SIAM Journal on Mathematical Analysis, 23 (1992) 1482-1518.

[38] B. Hassani, E. Hinton, A review of homogenization and topology optimization I-homogenization theory for media with periodic structure, Computers \& Structures, 69 (1998) 707-717.

[39] B. Hassani, E. Hinton, A review of homogenization and topology opimization II-analytical and numerical solution of homogenization equations, Computers \& Structures, 69 (1998) 719-738.

[40] B. Hassani, E. Hinton, A review of homogenization and topology optimization III-topology optimization using optimality criteria, Computers \& Structures, 69 (1998) 739-756.

[41] A. Bensoussan, J.-L. Lions, G. Papanicolaou, Asymptotic analysis for periodic structures, American Mathematical Soc., 2011.

[42] Y. Prawoto, Seeing auxetic materials from the mechanics point of view: A structural review on the negative Poisson's ratio, Computational Materials Science, 58 (2012) 140-153.

[43] S.J. Hollister, N. Kikuchi, A comparison of homogenization and standard mechanics analyses for periodic porous composites, Computational Mechanics, 10 (1992) 73-95.

[44] J.A. Sethian, A. Wiegmann, Structural Boundary Design via Level Set and Immersed Interface Methods, Journal of Computational Physics, 163 (2000) 489-528.

[45] S.J. Osher, F. Santosa, Level set methods for optimization problems involving geometry and constraints. I. frequencies of a two-density inhomogeneous drum, Journal of Computational Physics, 171 (2001) 272-288.

[46] G. Allaire, F. Jouve, A.-M. Toader, A level-set method for shape optimization, C. R. Acad. Sci. Paris, Serie I, 334 (2002) 1-6.

[47] M.Y. Wang, X.M. Wang, PDE-driven level sets, shape sensitivity, and curvature flow for structural topology optimization, Computer Modeling in Engineering \& Sciences, 6 (2004) 373-395.

[48] T. Belytschko, S.P. Xiao, C. Parimi, Topology optimization with implicitly function and regularization, interational Journal of Numerical Method for Engineering, 57 (2003) 1177-1196.

[49] S. Wang, M.Y. Wang, Radial basis functions and level set method for structural topology optimization, Interational Journal of Numerical Method for Engineering, 65 (2006a) 2060-2090.

[50] S. Wang, M.Y. Wang, Structural shape and topology optimization using implicit free boundary parameterization method, Computer Modeling in Engineering \& Sciences, 13 (2006b) 119-147.

[51] Z. Luo, M.Y. Wang, L. Tong, S. Wang, Shape and topology optimization of compliant mechanisms using a parameterization level set method, Journal of Computational Physics, 227 (2007) 680-705.

[52] S.O.R. Fedkiw, S. Osher, Level set methods and dynamic implicit surfaces, Surfaces, 44 (2002) 77.

[53] O. Sigmund, Design of multiphysics actuators using topology optimization-Part II: Two-material structures, Computer methods in applied mechanics and engineering, 190 (2001) 6605-6627.

[54] T. Gao, W. Zhang, P. Duysinx, Comparison of volume constraint and mass constraint in structural topology optimization with multiple materials, in: Book of abstracts and proceeding of the 2nd International Conference on Engineering Optimization, 2010.

[55] T. Gao, W. Zhang, A mass constraint formulation for structural topology optimization with multiphase materials, International Journal for Numerical Methods in Engineering, 88 (2011) 774-796.

[56] M.Y. Wang, X. Wang, "Color" level sets: a multi-phase method for structural topology optimization with multiple materials, Computer Methods in Applied Mechanics and Engineering, 193 (2004) 469-496.

[57] M.Y. Wang, X. Wang, Y. Mei, S. Chen, Design of multimaterial compliant mechanisms using level-set methods, Journal of mechanical design, 127 (2005) 941-956. 
[58] G. Allaire, F. Jouve, G. Michailidis, Structural and multi-functional optimization using multiple phases and a level-set method, (2013).

[59] J. Lie, M. Lysaker, X.-C. Tai, A variant of the level set method and applications to image segmentation, Mathematics of computation, 75 (2006) 1155-1174.

[60] P. Wei, M.Y. Wang, Piecewise constant level set method for structural topology optimization, International Journal for Numerical Methods in Engineering, 78 (2009) 379-402.

[61] H. Li, X.-C. Tai, Piecewise constant level set methods for multiphase motion, International Journal of Numerical Analysis and Modeling, 4 (2007) 291-305.

[62] B. Merriman, J.K. Bence, S.J. Osher, Motion of multiple junctions: A level set approach, Journal of Computational Physics, 112 (1994) 334-363.

[63] X. Zhang, J.-S. Chen, S. Osher, A multiple level set method for modeling grain boundary evolution of polycrystalline materials, Interaction and Multiscale Mechanics, 1 (2008) 178-191.

[64] S. Chen, S. Gonella, W. Chen, W.K. Liu, A level set approach for optimal design of smart energy harvesters, Computer Methods in Applied Mechanics and Engineering, 199 (2010) 2532-2543.

[65] G. Allaire, A. Craig, Numerical analysis and optimization, Oxford Univ. Press, UK, 2007.

[66] E.J. Haug, K.K. Choi, V. Komkov, Design sensitivity analysis of structural systems, Academic Press, Orlando, 1986.

[67] K.K. Choi, N.H. Kim, Structural Sensitivity Analysis and Optimization 1: Linear Systems, Springer, 2005.

[68] T. Belytschko, W.K. Liu, B. Moran, Nonlinear Finite Elements for Continua and Structures, John Wiley \& Sons Ltd., New York, 2000. 